\title{
THE CHANDRA X-RAY SURVEY OF PLANETARY NEBULAE (CHANPLANS): PROBING BINARITY, MAGNETIC FIELDS, AND WIND COLLISIONS
}

\author{
J. H. Kastner ${ }^{1}$, R. Montez JR. ${ }^{1}$, B. Balick ${ }^{2}$, D. J. Frew $^{3}$, B. Miszalski ${ }^{4}, 5$, R. SAhai ${ }^{6}$, E. Blackman ${ }^{7}$, Y.-H. Chu ${ }^{8}$, \\ O. De Marco ${ }^{3}$, A. Frank $^{7}$, M. A. Guerrero ${ }^{9}$, J. A. Lopez $^{10}$, V. Rapson ${ }^{1}$, A. Zijlstra ${ }^{11}$, E. Behar ${ }^{12}$, V. Bujarrabal ${ }^{13}$, \\ R. L. M. Corradi ${ }^{14,15}$, J. Nordhaus ${ }^{16,22}$, Q. A. PARKer ${ }^{3,17}$, C. SANDIN ${ }^{18}$, D. SChÖNBerner ${ }^{18}$, N. SOKer ${ }^{12}$, J. L. SOKOLOSKI ${ }^{19}$, \\ M. STEFFEN ${ }^{18}$, T. Ueta ${ }^{20}$, AND E. Villaver ${ }^{21}$ \\ ${ }^{1}$ Center for Imaging Science and Laboratory for Multiwavelength Astrophysics, Rochester Institute of Technology, 54 Lomb Memorial Drive, \\ Rochester, NY 14623, USA; jhk@ cis.rit.edu \\ ${ }^{2}$ Department of Astronomy, University of Washington, Seattle, WA, USA \\ ${ }^{3}$ Department of Physics and Astronomy and Macquarie Research Centre for Astronomy, Astrophysics and Astrophotonics, \\ Macquarie University, Sydney, NSW 2109, Australia \\ ${ }^{4}$ South African Astronomical Observatory, P.O. Box 9, Observatory, 7935, South Africa \\ ${ }^{5}$ Southern African Large Telescope Foundation, P.O. Box 9, Observatory, 7935, South Africa \\ 6 Jet Propulsion Laboratory, California Institute of Technology, MS 183-900, Pasadena, CA 91109, USA \\ ${ }^{7}$ Department of Physics and Astronomy, University of Rochester, Rochester, NY, USA \\ 8 Department of Astronomy, University of Illinois, Champagne-Urbana, IL, USA \\ ${ }^{9}$ Instituto de Astrofísica de Astronomía, Glorieta de la Astronomía s/n, Granada 18008, Spain \\ ${ }^{10}$ Instituto de Astronomia, Universidad Nacional Autonoma de Mexico, Campus Ensenada, Apdo. Postal 22860, Ensenada, B. C., Mexico \\ ${ }^{11}$ School of Physics and Astronomy, University of Manchester, Manchester M13 9PL, UK \\ ${ }^{12}$ Department of Physics, Technion, Israel; soker@physics.technion.ac.il \\ ${ }^{13}$ Observatorio Astronomico Nacional, Apartado 112, E-28803, Alcala de Henares, Spain \\ ${ }^{14}$ Instituto de Astrofísica de Canarias, E-38200 La Laguna, Tenerife, Spain \\ 15 Departamento de Astrofísica, Universidad de La Laguna, E-38206 La Laguna, Tenerife, Spain \\ ${ }^{16}$ Center for Computational Relativity and Gravitation, Rochester Institute of Technology, Rochester, NY 14623, USA \\ ${ }_{17}$ Australian Astronomical Observatory, P.O. Box 296, Epping, NSW 2121, Australia \\ ${ }^{18}$ Leibniz Institute for Astrophysics Potsdam (AIP), An der Sternwarte 16, D-14482 Potsdam, Germany \\ ${ }^{19}$ Columbia Astrophysics Laboratory, Columbia University, New York, NY 10027, USA \\ ${ }^{20}$ Department of Physics and Astronomy, University of Denver, Denver, CO 80208, USA \\ ${ }^{21}$ Departamento de Física Teórica, Universidad Autónoma de Madrid, Cantoblanco 28049 Madrid, Spain; eva.villaver@uam.es \\ Received 2012 April 13; accepted 2012 June 3; published 2012 July 12
}

\begin{abstract}
We present an overview of the initial results from the Chandra Planetary Nebula Survey (ChanPlaNS), the first systematic (volume-limited) Chandra X-Ray Observatory survey of planetary nebulae (PNe) in the solar neighborhood. The first phase of ChanPlaNS targeted 21 mostly high-excitation PNe within $\sim 1.5 \mathrm{kpc}$ of Earth, yielding four detections of diffuse $\mathrm{X}$-ray emission and nine detections of X-ray-luminous point sources at the central stars (CSPNe) of these objects. Combining these results with those obtained from Chandra archival data for all (14) other PNe within $\sim 1.5 \mathrm{kpc}$ that have been observed to date, we find an overall X-ray detection rate of $\sim 70 \%$ for the 35 sample objects. Roughly $50 \%$ of the PNe observed by Chandra harbor X-ray-luminous CSPNe, while soft, diffuse X-ray emission tracing shocks-in most cases, "hot bubbles"-formed by energetic wind collisions is detected in $\sim 30 \%$; five objects display both diffuse and point-like emission components. The presence (or absence) of X-ray sources appears correlated with PN density structure, in that molecule-poor, elliptical nebulae are more likely to display X-ray emission (either point-like or diffuse) than molecule-rich, bipolar, or Ring-like nebulae. All but one of the point-like CSPNe X-ray sources display X-ray spectra that are harder than expected from hot $(\sim 100 \mathrm{kK})$ central stars emitting as simple blackbodies; the lone apparent exception is the central star of the Dumbbell nebula, NGC 6853. These hard X-ray excesses may suggest a high frequency of binary companions to CSPNe. Other potential explanations include self-shocking winds or PN mass fallback. Most PNe detected as diffuse X-ray sources are elliptical nebulae that display a nested shell/halo structure and bright ansae; the diffuse $\mathrm{X}$-ray emission regions are confined within inner, sharp-rimmed shells. All sample PNe that display diffuse X-ray emission have inner shell dynamical ages $\lesssim 5 \times 10^{3} \mathrm{yr}$, placing firm constraints on the timescale for strong shocks due to wind interactions in PNe. The high-energy emission arising in such wind shocks may contribute to the high excitation states of certain archetypical "hot bubble" nebulae (e.g., NGC 2392, 3242, 6826, and 7009).
\end{abstract}

Key words: binaries: general - planetary nebulae: general - stars: AGB and post-AGB

Online-only material: color figures

\section{INTRODUCTION}

Planetary nebulae (PNe), the near endpoints of stellar evolution for intermediate-mass $\left(\sim 1-8 M_{\odot}\right)$ stars, have served

\footnotetext{
${ }^{22}$ NSF Astronomy and Astrophysics Fellow.
}

as astrophysical laboratories for more than a century (Aller 1956). With their relatively large numbers in close proximity $(\sim 200$ PNe lie within $\sim 2 \mathrm{kpc}$; Cahn et al. 1992; Frew 2008; Stanghellini et al. 2008), PNe serve as primary examples of plasma and shock processes and provide essential tests of theories of stellar evolution and the origin and enrichment of the 
heavy elements in the universe (Kwok 2000). PNe are best known as $\sim 10^{4} \mathrm{~K}$ optical emission line sources, yet many objects harbor cold $(<100 \mathrm{~K})$, dense $\left(\sim 10^{6}-10^{7} \mathrm{~cm}^{-3}\right)$ gas, and dust, and some of these same PNe also display emission from rarefied, hot $\left(T>10^{6} \mathrm{~K}\right), \mathrm{X}$-ray-emitting plasma. In principle, each of these temperature and density regimes informs us about the properties of the progenitor star system and its evolution.

Long thought to signify the transition of single stars from asymptotic giant branch (AGB) to white dwarf (WD) evolutionary stages, PNe exhibit a dazzling variety of optical and near-infrared morphologies: round; elliptical; bipolar; highly point symmetric; chaotic; and clumpy (e.g., Sahai et al. 2011 and references therein). The physical mechanisms responsible for this PN morphological menagerie-and, in particular, for the evident transformation from a quasi-isotropic wind during the progenitor star AGB phase to nonspherical or even highly collimated outflow during the PN phase-have been the subject of intense interest and hot debate among PN researchers over the past two decades (e.g., Balick \& Frank 2002; Zijlstra et al. 2011; Kastner 2011). At the heart of this debate lies the question: Do some, most, or even all PNe actually represent the ejection and photoionization of the envelopes of AGB stars in binary systems? While stellar magnetic fields generated within the AGB progenitor may play a role in the early shaping of $\mathrm{PNe}$ (Blackman et al. 2001a and references therein), the large fraction of PNe that display nonspherical geometry (Soker 1997; Sahai \& Trauger 1998) - and the fact that at least $\sim 20 \%$ of $\mathrm{PNe}$ are known to have binary companions to their central stars (Bond 2000; Miszalski et al. 2009a)_indeed suggests that a significant fraction of $\mathrm{PNe}$ represent the products of interacting binary star systems, within which preferred symmetry axes are found (e.g., De Marco 2009; Miszalski et al. 2009b; Jones et al. 2012 and references therein).

A binary companion to the PN central star (hereafter CSPN) can influence the PN shape in various ways, e.g., via formation of an accretion disk around the secondary (e.g., Morris 1987; Mastrodemos \& Morris 1998; Soker \& Rappaport 2000) or angular momentum injection (during, e.g., a common envelope phase) and the consequent generation of a disk and/or a strong magnetic dynamo at the primary (e.g., Reyes-Ruiz \& López 1999; Nordhaus \& Blackman 2006; Nordhaus et al. 2007). These models have recently received strong observational support, in the form of examples of close binary CSPNe that drive jets (e.g., Corradi et al. 2011; Miszalski et al. 2011). The potential role of disks as agents of PN outflow collimation, via jet formation (e.g., Soker \& Livio 1994; Blackman et al. 2001b), invites analogies to the disk/jet connection in, e.g., young stellar objects (Kastner 2009). There also appears to be a close link between symbiotic binary systems and both "butterfly" (bipolar) PNe (Corradi \& Schwarz 1995) and high-[O III]-luminosity PNe (Frankowski \& Soker 2009); the latter class of PN serves as an extragalactic standard candle (Ciardullo et al. 2005).

Standard models for the formation of PNe (e.g., Kwok et al. 1978; Schmidt-Voigt \& Koeppen 1987; Marten \& Schönberner 1991; Villaver et al. 2002; Perinotto et al. 2004) predict that the fast ( $v_{w} \simeq 500-1500 \mathrm{~km} \mathrm{~s}^{-1}$ ) wind emanating from the pre-WD at the core of the PN rams into the previously expelled AGB envelope (which was ejected at $\sim 10 \mathrm{~km} \mathrm{~s}^{-1}$ ), thereby sweeping the AGB ejecta into a thin shell and shocking the fast wind to temperatures $\gg 10^{6} \mathrm{~K}$. Such wind interaction models therefore predict that $\mathrm{PNe}$ should harbor X-ray-luminous, evacuated bubbles (e.g., Zhekov \& Perinotto 1996; Akashi et al. 2006; Steffen et al. 2008; Lou \& Zhai 2010). Over the past decade,
X-ray imaging by Chandra and XMM-Newton has provided compelling observational evidence for such CSPN-wind-blown "hot bubbles" (Kastner et al. 2008, and references therein). About a dozen PNe previously targeted by the two contemporary $\mathrm{X}$-ray observatories have been detected as diffuse X-ray sources (Kastner et al. 2000, 2001, 2003; Chu et al. 2001; Guerrero et al. 2002, 2005; Montez et al. 2005; Gruendl et al. 2006).

Chandra imaging has also revealed that certain PNe harbor $\mathrm{X}$-ray point sources at their cores, with source X-ray spectral energy distributions (SEDs) that cannot be explained as the Wien tails of CSPNe emitting as simple hot blackbodies (e.g., Guerrero et al. 2001; Kastner et al. 2003; Montez et al. 2010). Notably - when imaged by Chandra - a few PNe reveal both soft, diffuse and harder, point-like X-ray emission (e.g., NGC 6543; Chu et al. 2001). These two "flavors" of PN X-ray sources-diffuse and point-like-serve as complementary probes of the mechanisms underlying PN structural evolution.

Diffuse X-ray sources. Several trends have emerged from the examples of diffuse PN X-ray sources detected to date by Chandra and XMM (Kastner 2007; Kastner et al. 2008 and references therein).

1. Those PNe in which X-ray-luminous "hot bubbles" have been detected thus far all harbor CSPNe that drive particularly energetic winds (speeds $V_{W} \sim 1000 \mathrm{~km} \mathrm{~s}^{-1}$ at mass-loss rates $\dot{M} \gtrsim$ a few $\times 10^{-8} M_{\odot} \mathrm{yr}^{-1}$ ). A disproportionate fraction of these CSPNe are of the relatively rare Wolf-Rayet (WR) type (with $\dot{M} \gtrsim 10^{-6} M_{\odot} \mathrm{yr}^{-1}$ ).

2. Hot bubble X-ray luminosity seems to be weakly correlated with present-day CSPN wind luminosity $L_{w}=(1 / 2) \dot{M} v_{w}^{2}$ and anticorrelated with bubble radius, indicative of the close connection between the evolution of CSPN winds and PN hot bubbles (a connection explored in various theoretical investigations; e.g., Akashi et al. 2006, 2007; Steffen et al. 2008).

3. In cases in which hot bubble X-ray emission is detected, the optical/IR structures that enclose the regions of diffuse $\mathrm{X}$-rays have thin, bright, uninterrupted edges, suggesting that the diffuse X-ray-emitting gas is spatially confined and therefore inhibited from expanding adiabatically.

Among the more surprising results obtained from X-ray imaging spectroscopy of PNe are the low temperatures of the shocked (X-ray-emitting) wind gas $\left(T_{X}\right)$ in PN hot bubbles. Diffuse X-ray emission regions within PNe typically display $T_{X}$ in the narrow range $\sim 1-2 \mathrm{MK}$, which is one to two orders of magnitude lower than expected, based on simple jump conditions, for central star wind speeds $V_{W} \sim 1000 \mathrm{~km} \mathrm{~s}^{-1}$; furthermore, hot bubble $T_{X}$ does not appear to depend on CSPN wind velocity (Kastner et al. 2008; Montez 2010). Many temperature regulation mechanisms have been proposed to explain these results (see Soker \& Kastner 2002; Stute \& Sahai 2006; Kastner et al. 2008; Soker et al. 2010 and references therein). The low observed values of $T_{X}$ may indicate that hot bubble physical conditions are established during early phases of the post-AGB/pre-PN evolution of central stars with rapidly evolving winds (Akashi et al. 2006, 2007). Alternatively, an active temperature-moderating mechanism may govern the observed $T_{X}$. Potential mechanisms include heat conduction (Steffen et al. 2008; Li et al. 2012), mixing of nebular and fast wind material (Chu et al. 2001), or a small mass of "pickup ions" that wander into the hot bubble from cold, neutral nebular clumps (analogous to a mechanism proposed to cool the solar wind; see Soker et al. 2010). The heat conduction 
models appear to hold particular promise (Steffen et al. 2008; Montez 2010; C. Sandin et al. 2012, in preparation). On the other hand, measurements of the elemental abundances within the X-ray-emitting plasma of $\mathrm{BD}+30^{\circ} 3639$ - the most luminous diffuse X-ray PN and, hence, the only object for which such precise hot bubble abundance and temperature determinations are presently available (via Chandra X-ray gratings spectroscopy; Yu et al. 2009) — closely match that of its [WC]-type central star. This strong resemblance led Yu et al. (2009) to conclude that the superheated plasma within BD $+30^{\circ} 3639$ consists of "pure" fast wind material, such that neither heat conduction nor mixing likely plays an important role in determining its (low) characteristic $T_{X}$ of $\sim 2 \times 10^{6} \mathrm{~K}$.

$X$-ray point sources at CSPNe. Einstein and ROSAT established that certain high-excitation $\mathrm{PNe}$ harbor soft X-ray sources indicative of emission from hot ( $\gtrsim 100 \mathrm{kK})$ CSPN photospheres (e.g., Motch et al. 1993; Guerrero et al. 2000). However, Chandra imaging has revealed intriguing examples of X-ray sources at CSPNe that are too hard to be modeled in terms of simple blackbody emission from a pre-WD stellar photosphere (Guerrero et al. 2001; Hoogerwerf et al. 2007; Montez et al. 2010). The Helix Nebula (NGC 7293) central star is perhaps the best-characterized example of such a "hard X-ray excess" source (Guerrero et al. 2001). Among the thousands of WDs that have been observed (mostly serendipitously) by $X M M$ or ROSAT, only a handful of isolated, supposedly single WDs-including the post-PN object PG 1159-display similarly hard spectra (Bilíková et al. 2010). Via analogy with cataclysmic variables and symbiotic binaries, this new class of relatively hard PN X-ray point source may be hinting at the presence of binary companions and/or accretion processes associated with CSPNe (Kastner 2007). The hard X-rays may arise from accretion onto a compact, hot companion (Kastner et al. 2003), or from the corona of a late-type companion that has been "rejuvenated" via accretion of pre-PN (AGB) wind material (Jeffries \& Stevens 1996; Soker \& Kastner 2002), as appears to be the case for the PNe DS 1, HFG 1, LoTr 5 (all of which are known binaries; Montez et al. 2010), and K 1-6 (a nearby, binary CSPN that was detected by ROSAT; Frew et al. 2011). Other (non-binary) models also could explain the presence of "hard excess" point sources, however, such as internal wind shocks analogous to those observed in O stars (Guerrero et al. 2001) or mass infall, e.g., from a residual, Kuiper-Belt-like debris disk orbiting the CSPN (Su et al. 2007; Bilíková et al. 2010).

The X-ray emission characteristics of PNe just described have been assembled from piecemeal and uncoordinated Chandra programs, each of which targeted perhaps one or two objects. The resulting small number statistics, combined with the haphazard nature of the sample of PNe observed thus far in X-rays at Chandra's subarcsecond spatial resolution-which is required to distinguish between point-like and diffuse emission-leaves many fundamental questions unanswered: under what circumstances do wind-wind shocks lead to hot bubbles within PNe, and how do these hot bubbles evolve with time? How are the kinematics of PNe and the wind properties of their central stars related to the luminosity and morphology of PN X-ray emission? What heating and cooling mechanisms govern the temperatures of the X-ray-emitting plasmas within PNe? How might detection and characterization of X-ray point sources at CSPNe improve our knowledge of the frequency and characteristics of binary systems within PNe, and the relationships of such binaries to potentially related systems such as symbiotic stars and SN Ia progenitor binaries?
To address these questions, we are undertaking the Chandra Planetary Nebula Survey (CHANPlaNS) - a Chandra X-Ray Observatory survey of the $\sim 120$ known PNe within $\sim 1.5 \mathrm{kpc}$ of Earth (as drawn from the comprehensive catalogs compiled by Acker et al. 1992; Cahn et al. 1992; Frew 2008). ChanPlaNS constitutes one element of a planned comprehensive observational and theoretical campaign to understand the shaping of PNe, as described in the "Rochester White Paper" (De Marco et al. 2011). The ChanPlaNS survey began with a 570 ks Chandra Cycle 12 Large Program targeting 21 (mostly high-excitation) PNe from among this sample, thereby roughly doubling the number of PNe within $\sim 1.5 \mathrm{kpc}$ that have been observed by Chandra. In this paper, we describe initial results obtained for this, the first statistically significant, volumelimited sample of PNe to be imaged in X-rays at high spatial resolution.

\section{SAMPLE SELECTION, OBSERVATIONS, AND DATA REDUCTION}

\subsection{Planetary Nebulae within $\sim 1.5 \mathrm{kpc}$ Observed by Chandra}

The sample of $21 \mathrm{PN}$ targeted during Chandra Cycle 12 was assembled from the comprehensive lists of well-studied PNe in Gurzadian (1988), Acker et al. (1992), and Frew (2008). Most of the targeted PNe are "high-excitation" objects, characterized by bright lines of highly ionized species of, e.g., He, C, N, O, and $\mathrm{Ne}$ that are generally indicative of central stars with high effective temperatures (i.e., $T_{\text {eff }} \gtrsim 10^{5} \mathrm{~K}$ ). We initially selected those PNe for which Gurzadian (1988) lists $I(\lambda 4686) / I(\mathrm{H} \beta)$ $\gtrsim 0.15$, corresponding (in principle) to $T_{\text {eff }} \gtrsim 10^{5} \mathrm{~K}$. To limit our targets to the subset of high-excitation PNe that are closest to Earth, we restricted the Cycle 12 target list to objects with (1) mean distances $D \leqslant 1.5 \mathrm{kpc}$ based on data compiled in Acker et al. (1992), and (2) distances $D \leqslant 1.5 \mathrm{kpc}$ according to either Cahn et al. (1992) or Frew (2008). To this list of objects from Gurzadian (1988), we added the PN Longmore 16 (hereafter Lo 16; Longmore 1977; D. Frew et al. 2012, in preparation).

Based on a search of the Chandra archives, we identify 14 additional PNe with $D \leqslant 1.5 \mathrm{kpc}$ previously observed by Chandra (13 targeted, and 1 serendipitously observed). In contrast to the volume-limited, excitation-selected Cycle 12 ChanPlanS sample, these $14 \mathrm{PNe}$ were targeted for a variety of (unrelated) reasons-e.g., pre-Chandra (e.g., ROSAT) X-ray detections (Kastner et al. 2000; Chu et al. 2001; Guerrero et al. 2001), evidence for rapid structural evolution (Kastner et al. 2001), and binary or WR-type central stars (Montez et al. 2005, 2010).

The full (Cycle 12 plus archival data) sample of $35 \mathrm{PNe}$ within $\sim 1.5 \mathrm{kpc}$ observed by Chandra is listed in Table $1 .^{23}$ This table summarizes basic PN and CSPN data for the sample objects; these data are mainly compiled from Frew (2008), with additional morphological classifications following the system described in Sahai et al. (2011, see their Table 2) and results from available molecular $\left(\mathrm{H}_{2}\right)$ line observations from Kastner et al. (1996). The last column of Table 1 specifies whether or not the Chandra observations (Sections 2.2.1 and 2.3) resulted

\footnotetext{
23 Subsets of the sample listed in Table 1 are the subjects of Herschel Space Observatory studies of the far-IR emission properties of PNe: "Mass loss of Evolved Stars," PI: M. Groenewegen, and "The Herschel Planetary Nebula Survey," PI: T. Ueta (early results appear in van Hoof et al. 2011; T. Ueta et al. 2012 , in preparation, respectively).
} 
Table 1

Planetary Nebulae Within $1.5 \mathrm{kpc}^{\mathrm{a}}$ Observed by Chandra

\begin{tabular}{|c|c|c|c|c|c|c|c|c|c|c|}
\hline Name & PN G & $\begin{array}{c}\text { Morph. }^{\mathrm{b}} \\
\text { (F08/SMV11) }\end{array}$ & $\begin{array}{c}D \\
(\mathrm{kpc})\end{array}$ & $\begin{array}{c}R \\
(\mathrm{pc})\end{array}$ & $\begin{array}{c}\text { Age } \\
\left(10^{3} \mathrm{yr}\right)\end{array}$ & $\begin{array}{c}T_{\star} \\
(\mathrm{kK})\end{array}$ & Sp. Type & Comp. & $\mathrm{H}_{2}{ }^{\mathrm{c}}$ & 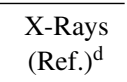 \\
\hline \multicolumn{11}{|c|}{ PNe observed in Cycle 12} \\
\hline NGC 650 (M 76) & $130.9-10.5$ & $\mathrm{Bas}(\mathrm{h}) / \mathrm{Bcbpa}$ & 1.20 & 0.40 & 10 & 140 & PG1159 & $\ldots$ & Y & $\mathrm{N}$ \\
\hline NGC 1360 & $220.3-53.9$ & Efp/Ecs & 0.38 & 0.31 & 9 & 110 & $\mathrm{O}(\mathrm{H})$ & $\ldots$ & $\mathrm{N}$ & $\mathrm{P}$ \\
\hline NGC 1514 & $165.5-15.2$ & Ems/Is & 0.37 & 0.12 & 5 & 60 & $\mathrm{O}(\mathrm{H}):$ & A0III & $\ldots$ & $\mathrm{P}$ \\
\hline NGC 2346 & $215.6+03.6$ & Bs/Bobsp & 0.90 & 0.14 & 11 & $\geqslant 80$ & $\mathrm{O}(\mathrm{H}) ?$ & $\mathrm{~A} 5 \mathrm{~V}$ & $\mathrm{Y}$ & $\mathrm{N}$ \\
\hline NGC 2371 & $189.1+19.8$ & Eps/Bcbspa & 1.41 & 0.13 & 3 & 100 & {$[\mathrm{WO} 1]^{\mathrm{e}}$} & $\ldots$ & $\mathrm{N}$ & $\mathrm{D}, \mathrm{P}$ \\
\hline NGC 2438 & $231.8+04.1$ & $\operatorname{Emr}(\mathrm{h}) / \operatorname{Ecs}$ & 1.42 & 0.27 & 12 & 124 & $\operatorname{hgO}(\mathrm{H})$ & $\ldots$ & $\ldots$ & $\mathrm{N}$ \\
\hline NGC 3242 & $261.0+32.0$ & Em(h)/Ecspaih & 1.00 & 0.10 & 4 & 89 & $\mathrm{O}(\mathrm{H})$ & $\ldots$ & $\mathrm{N}$ & $\mathrm{D}$ \\
\hline NGC 3587 (M 97) & $148.4+57.0$ & Rfm:/Rspi & 0.76 & 0.38 & 11 & 105 & $\operatorname{hgO}(\mathrm{H})$ & $\ldots$ & $\mathrm{N}$ & $\mathrm{N}$ \\
\hline NGC 6302 & $349.5+01.0$ & Bps/Btp & 1.17 & 0.16 & $\ldots$ & 220 & $\ldots$ & $\ldots$ & $\mathrm{Y}$ & $\mathrm{N}$ \\
\hline NGC 6445 & $008.0+03.9$ & $\mathrm{Bs} / \mathrm{Mpi}$ & 1.39 & 0.14 & 3 & 170 & $\ldots$ & $\ldots$ & $\mathrm{Y}$ & $\mathrm{P}$ \\
\hline NGC 6720 (M 57) & $063.1+13.9$ & $\operatorname{Ebmr}(\mathrm{h}) / \operatorname{Ecsh}$ & 0.70 & 0.13 & 6 & 148 & $\operatorname{hgO}(\mathrm{H})$ & $\ldots$ & $\mathrm{Y}$ & $\mathrm{N}$ \\
\hline NGC 6772 & $033.1-06.3$ & $\mathrm{Ep} / \mathrm{E}$ & 1.20 & 0.22 & 19 & 135 & $\ldots$ & $\ldots$ & $\mathrm{Y}$ & $\mathrm{N}$ \\
\hline NGC 6781 & $041.8-02.9$ & $\operatorname{Bam}(\mathrm{h}:) / \mathrm{Bth}$ & 0.95 & 0.32 & 26 & 112 & DAO & $\ldots$ & $\mathrm{Y}$ & $\mathrm{N}$ \\
\hline NGC 6804 & $045.7-04.5$ & Eam/Ms & 1.47 & 0.19 & 7 & 85 & $\mathrm{O}(\mathrm{H})$ & $\mathrm{dM} ?^{\mathrm{f}}$ & Y & $\mathrm{N}$ \\
\hline NGC 6853 (M 27) & $060.8-03.6$ & $\operatorname{Ebm}(\mathrm{h}) / \mathrm{Bbpih}$ & 0.38 & 0.37 & 11 & 135 & DAO & $\mathrm{dM} ?$ & $\mathrm{Y}$ & $\mathrm{P}$ \\
\hline NGC 7008 & $093.4+05.4$ & $\mathrm{Efp} / \mathrm{Bs}$ & 0.70 & 0.15 & 4 & 97 & $\mathrm{O}(\mathrm{H})$ & dG: & $\ldots$ & $\mathrm{P}$ \\
\hline NGC 7009 & $037.7-34.5$ & $\operatorname{Emps}(\mathrm{h}) / \mathrm{Lbspa}$ & 1.45 & 0.09 & 3 & 87 & $\mathrm{O}(\mathrm{H})$ & $\ldots$ & $\mathrm{N}$ & $\mathrm{D}, \mathrm{P}$ \\
\hline NGC 7094 & $066.7-28.2$ & Ras/Rs & 1.39 & 0.34 & 8 & 110 & PG1159 & $\ldots$ & $\mathrm{N}$ & $\mathrm{P}$ \\
\hline NGC 7662 & $106.5-17.6$ & $\operatorname{Emp}(\mathrm{h}) /$ Esah & 1.26 & 0.09 & 3 & 111 & $\mathrm{O}(\mathrm{H})^{\mathrm{g}}$ & $\ldots$ & $\mathrm{N}$ & $\mathrm{D}$ \\
\hline A 33 & $238.0+34.8$ & $\mathrm{Ra} / \mathrm{R}^{*}$ & 1.16 & 0.78 & 24 & 100 & $\mathrm{O}(\mathrm{H})$ & $\mathrm{dK} 3$ & $\ldots$ & $\mathrm{N}$ \\
\hline Lo 16 & $349.3-04.2$ & Eps/Ispa & 0.84 & 0.17 & $\ldots$ & $\geqslant 82$ & $\mathrm{O}(\mathrm{H})^{\mathrm{f}}$ & $\mathrm{dK} ?$ & $\ldots$ & $\mathrm{P} ?$ \\
\hline \multicolumn{11}{|c|}{ PNe with available archival data } \\
\hline NGC 40 & $120.0+09.8$ & $\operatorname{Eas}(\mathrm{h}) / \mathrm{Bbsh}$ & 1.02 & 0.11 & 4 & 48 & [WC8] & $\ldots$ & $\mathrm{N}$ & $\mathrm{D}(1)$ \\
\hline NGC 246 & $118.8-74.7$ & $\mathrm{Ea} / \mathrm{Es}$ & 0.50 & 0.29 & 8 & 140 & PG1159 & $\mathrm{K} 0 \mathrm{~V}$ & $\mathrm{~N}$ & $\mathrm{P}(2)$ \\
\hline NGC 2392 & $197.8+17.3$ & Rm/Rsai & 1.28 & 0.14 & 3 & 47 & $\mathrm{Of}(\mathrm{H})$ & $\mathrm{dM} ?$ & $\mathrm{~N}$ & $\mathrm{D}, \mathrm{P}(\mathrm{u})$ \\
\hline NGC 3132 & $272.1+12.3$ & $\mathrm{Er} / \mathrm{Mtsp}$ & 0.81 & 0.13 & 6 & 100 & $\ldots$ & A2IV-V & $\mathrm{Y}$ & $\mathrm{N}(\mathrm{u})$ \\
\hline NGC 4361 & $294.1+43.6$ & Es/Ebs & 0.95 & 0.27 & 8 & 126 & $\mathrm{O}(\mathrm{H})$ & $\ldots$ & $\mathrm{N}$ & $\mathrm{P}(\mathrm{u})$ \\
\hline NGC 6543 & $096.4+29.9$ & $\operatorname{Emps}(\mathrm{h}) / \mathrm{Mcspa}$ & 1.50 & 0.09 & 5 & 48 & Of-WR(H) ${ }^{\mathrm{h}}$ & $\ldots$ & $\mathrm{N}$ & $\mathrm{D}, \mathrm{P}(3,4)$ \\
\hline NGC 6826 & $083.5+12.7$ & $\operatorname{Emp}(\mathrm{h}) /$ Ecsah & 1.30 & 0.08 & 5 & 50 & $\mathrm{O} 3 \mathrm{f}(\mathrm{H})$ & $\ldots$ & $\mathrm{N}$ & $\mathrm{D}, \mathrm{P}(\mathrm{u})$ \\
\hline NGC 7027 & $084.9-03.4$ & Bs/Mctspih & 0.89 & 0.03 & 1.4 & 175 & $\ldots$ & $\ldots$ & $\mathrm{Y}$ & D (5) \\
\hline NGC 7293 & $036.1-57.1$ & Bams(h)/Ltspir & 0.22 & 0.46 & 21 & 110 & DAO & $\ldots$ & $\mathrm{Y}$ & $\mathrm{P}(4)$ \\
\hline $\mathrm{BD}+30^{\circ} 3639$ & $064.7+05.0$ & Er/Ecsarh & 1.30 & 0.02 & 1 & 32 & [WC9] & $\ldots$ & $\mathrm{Y}$ & $\mathrm{D}(6)$ \\
\hline DS 1 & $283.9+09.7$ & Efp/Is & 0.73 & 0.59 & 19 & 90 & $\mathrm{O}(\mathrm{H})$ & M5-6 V ${ }^{i}$ & $\ldots$ & $\mathrm{P}(7)$ \\
\hline HFG 1 & $136.3+05.5$ & $\mathrm{Ea}(\mathrm{h}:) / \mathrm{R}$ & 0.60 & 0.79 & 51 & 100 & $\mathrm{O}(\mathrm{H})$ & dG: & $\ldots$ & $P(7)$ \\
\hline IC 418 & $215.2-24.2$ & Em(h:)/Ecspih & 1.20 & 0.04 & 3 & 38 & $\mathrm{Of}(\mathrm{H})$ & $\ldots$ & $\mathrm{N}$ & $\mathrm{D}(\mathrm{u})$ \\
\hline LoTr 5 & $339.9+88.4$ & Eabf/Ss & 0.50 & 0.64 & 20 & 100 & $\mathrm{O}(\mathrm{H})$ & G5III & $\ldots$ & $P(7)$ \\
\hline
\end{tabular}

Notes.

${ }^{a}$ PN and central star data compiled from Frew (2008 and references therein) unless otherwise indicated.

${ }^{b}$ Morphologies as listed in Frew (2008, F08): B: bipolar, E: elliptical, R: round, a: asymmetry present, b: bipolar core present, f: filled (amorphous) center, m: multiple shells present, p: point symmetry present, r: ring structure dominant, s: internal structure noted, (h): distinct outer halo. Morphologies following an abbreviated and very slightly modified version of the classification system described in Sahai et al. (2011) (SMV11; see their Table 2): B: bipolar, M: multipolar, E: elongated, I: irregular, R: round, L: collimated lobe pair, S: spiral arm, c: closed outer lobes, o: open outer lobes; s: CSPN apparent, b: bright (barrel-shaped) central region, t: bright central toroidal structure; p: point symmetry, a: ansae, i: inner bubble, h: halo; r: radial rays.

c "Y" = near-IR $H_{2}$ detected; "N" = near-IR H $\mathrm{H}_{2}$ not detected; Kastner et al. (1996 and references therein).

d X-ray results key: $\mathrm{P}=$ point source; $\mathrm{D}=$ diffuse source; $\mathrm{N}=$ not detected. References to Chandra data obtained previous to Cycle 12: (1) Montez et al. 2005; (2) Hoogerwerf et al. 2007; (3) Chu et al. 2001; (4) Guerrero et al. 2001; (5) Kastner et al. 2001; (6) Kastner et al. 2000 (7) Montez et al. 2010; (u) unpublished archival data.

e Acker \& Neiner (2003).

${ }^{f}$ Frew, unpublished data.

g Herald \& Bianchi (2011).

h Mendez et al. (1990).

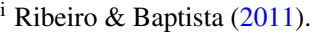

in the detection of point and/or diffuse X-ray sources for a given PN. These results are described in detail in Section 3. Results obtained from archival Chandra observations of four PNe (NGC 2392, 3132, 6826, and IC 418) are presented here for the first time (detailed analyses of the observations of NGC 2392, 6826, and IC 418 will appear in N. Ruiz et al. 2012, in preparation; M. Guerrero et al. 2012, in preparation).
Some characteristics of the present sample of PNe, relative to the general population of PNe in the solar neighborhood, are illustrated in Figure 1. The generally high-excitation states of the sample PNe (a result of the selection criteria applied to target objects in Cycle 12) are readily evident in their overall large ratios of [O III] to $\mathrm{H} \beta$ flux (with the notable exceptions of the low-excitation PNe IC 418, BD +30³639, and NGC 40). 

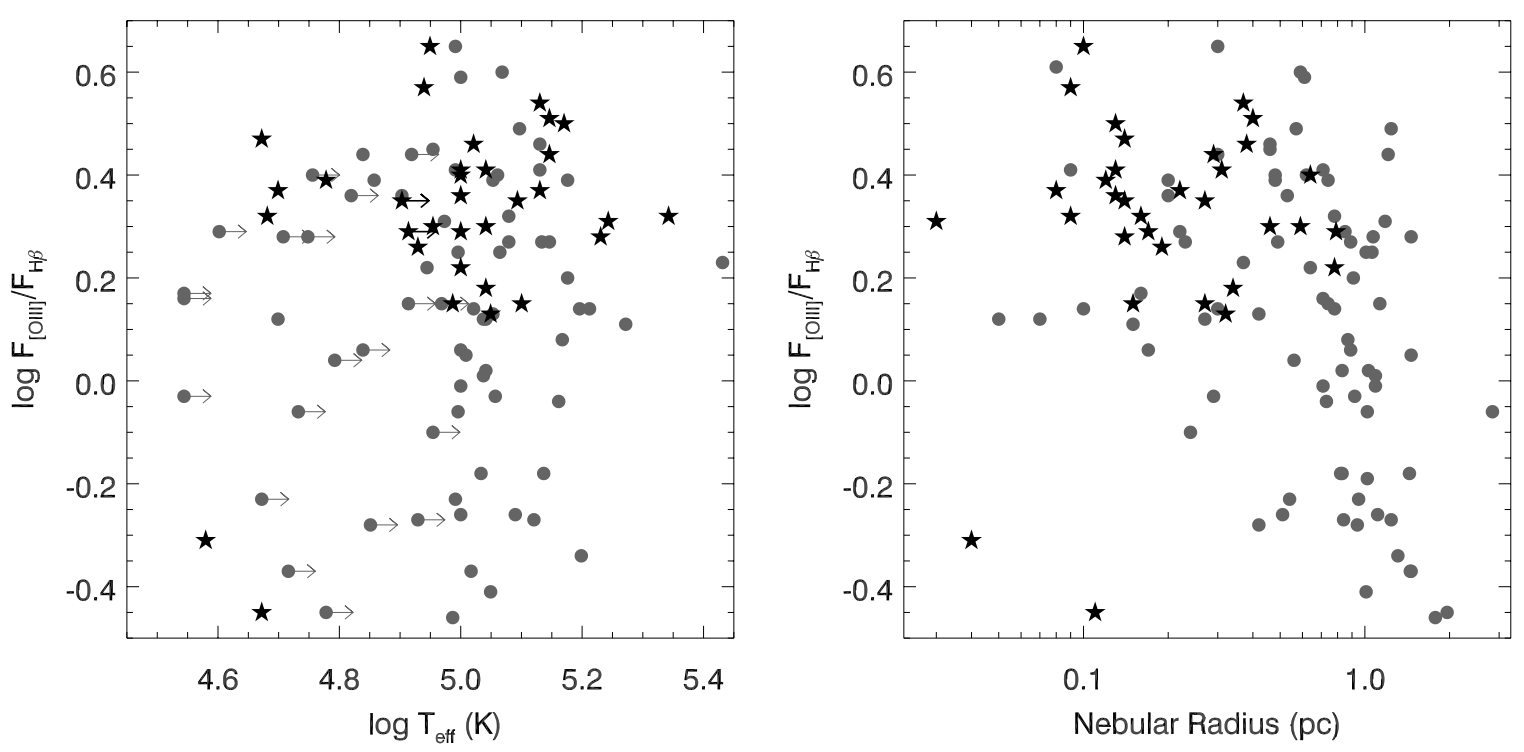

Figure 1. Characteristics of the sample of $35 \mathrm{PNe}$ within $1.5 \mathrm{kpc}$ observed in X-rays by Chandra (black stars) relative to the other $\sim 85 \mathrm{known}$ PNe within $1.5 \mathrm{kpc}$ (gray circles; distances from Frew 2008). Left: ratio of [O III] to $\mathrm{H} \beta$ fluxes vs. PN central star effective temperature. Right: [O III] to H $\beta$ flux ratio vs. nebular radius. The very low excitation, compact objects BD +303639 (a Chandra diffuse X-ray source; Kastner et al. 2000) and M 1-26 (not yet observed in X-rays) lie well below the range of $[\mathrm{O} \mathrm{III}]$ to $\mathrm{H} \beta$ flux ratio represented in these plots.

In addition, as expected, the sample includes many PNe with high $T_{\text {eff }}$ central stars (Figure 1, left panel), although about a third (including the three aforementioned low-excitation PNe) have estimated $T_{\text {eff }}$ in the range $30-100 \mathrm{kK}$ (most of the CSPN $T_{\text {eff }}$ values listed in Table 1 under column heading $T_{\text {star }}$ were obtained via the Zanstra method; Frew 2008).

\subsection{Observations}

\subsubsection{Chandra X-Ray Observatory}

All sample PNe were observed with Chandra's Advanced CCD Imaging Spectrometer (ACIS) using its primary backilluminated (BI) CCD (S3). With the exception of the PN LoTr 5 (which was observed serendipitously; Montez et al. 2010), each target PN was positioned at the nominal aim point of S3. Chandra/ACIS-S3 has energy sensitivity of $\sim 0.3-8 \mathrm{keV}$, with a field of view of $\sim 8^{\prime} \times 8^{\prime}$ and pixel size $0^{\prime \prime} .492$. In most observations, additional ACIS CCDs were active, extending the effective field of view; these data are not relevant to the analysis described here, however. Use of the BI CCD S3 provides soft X-ray sensitivity that is superior to that of the front-illuminated CCDs on ACIS, effectively extending energy sensitivity to $\sim 0.2 \mathrm{keV}$ for the softest PN sources (Section 3.2.1). In additiondue to the large fraction of photon events in which X-ray photon charge is split among adjacent pixels, in BI devices-use of S3 facilitates subpixel event repositioning (SER) in downstream processing (Section 2.3.1), such that post-SER ACIS-S3 images better sample the ( $\sim 0^{\prime} .5$ FWHM) core of the point-spread function produced by Chandra's High Resolution Mirror Assembly (Li et al. 2004). Observation IDs, dates, and exposure times are listed in Table 2 (all Cycle 12 Large Program observation identifiers begin with "12," apart from one short, followup exposure targeting NGC 6302).

\subsubsection{Ground-based Optical Imaging}

Images of a subset of Table 1 PNe (included in some panels of Figure 2; see Section 2.3) were obtained with the
Wisconsin-Indiana-Yale-NOAO (WIYN) $0.9 \mathrm{~m}$ telescope. ${ }^{24}$ The WIYN 0.9 m images were obtained in 2010 November with the S2KB CCD camera (0'. 6 pixels; 20 '.48 $\times 20$ '.48 field of view) and $\mathrm{H} \alpha$ filter. Exposure times ranged from $100 \mathrm{~s}$ to $500 \mathrm{~s}$, and images were subject to standard processing (dark subtraction, flat-fielding) and astrometric calibration. The $\mathrm{H} \alpha$ image of Lo 16 (exposure time $180 \mathrm{~s}$ ) used in Figure 2 was obtained with GMOS (Hook et al. 2004) on the Gemini $8 \mathrm{~m}$ telescope (as part of program GS-2009A-Q-35; see Miszalski et al. 2009b). The image of DS 1 is taken from the SuperCOSMOS H $\alpha$ Survey (Parker et al. 2005).

\subsection{Data Reduction: The CHANPLANS Pipeline}

\subsubsection{Reprocessing}

To generate a uniform set of high-level X-ray data products (i.e., images, source lists, spectra, and light curves) from the CHANPLANS observations, we have constructed a processing pipeline consisting of scripts that utilize both $\mathrm{CIAO}^{25}$ (version 4.3) tools and custom code. The first step is to reprocess the primary and secondary (event and ancillary data) data files provided by the Chandra X-ray Center. Reprocessing is performed with the CIAO chandra_repro script, which applies the latest calibrations available (CALDB version 4.4.6, in the case of the data presented here) and generates new observation data files (events, bad pixels, aspect solution, etc.). Reprocessing also includes application of SER, so as to optimize the spatial resolution of Chandra/ACIS-S3 (Li et al. 2004).

\subsubsection{Source Detection}

For each observation, we search for sources in seven energy filter bands using the CIAO wavelet-based source detection task

\footnotetext{
24 The $0.9 \mathrm{~m}$ telescope is operated by WIYN Inc. on behalf of a consortium of partner Universities and Organizations that includes RIT. WIYN is a joint partnership of the University of Wisconsin at Madison, Indiana University, Yale University, and the National Optical Astronomical Observatory.

25 http://cxc.harvard.edu/ciao/
} 

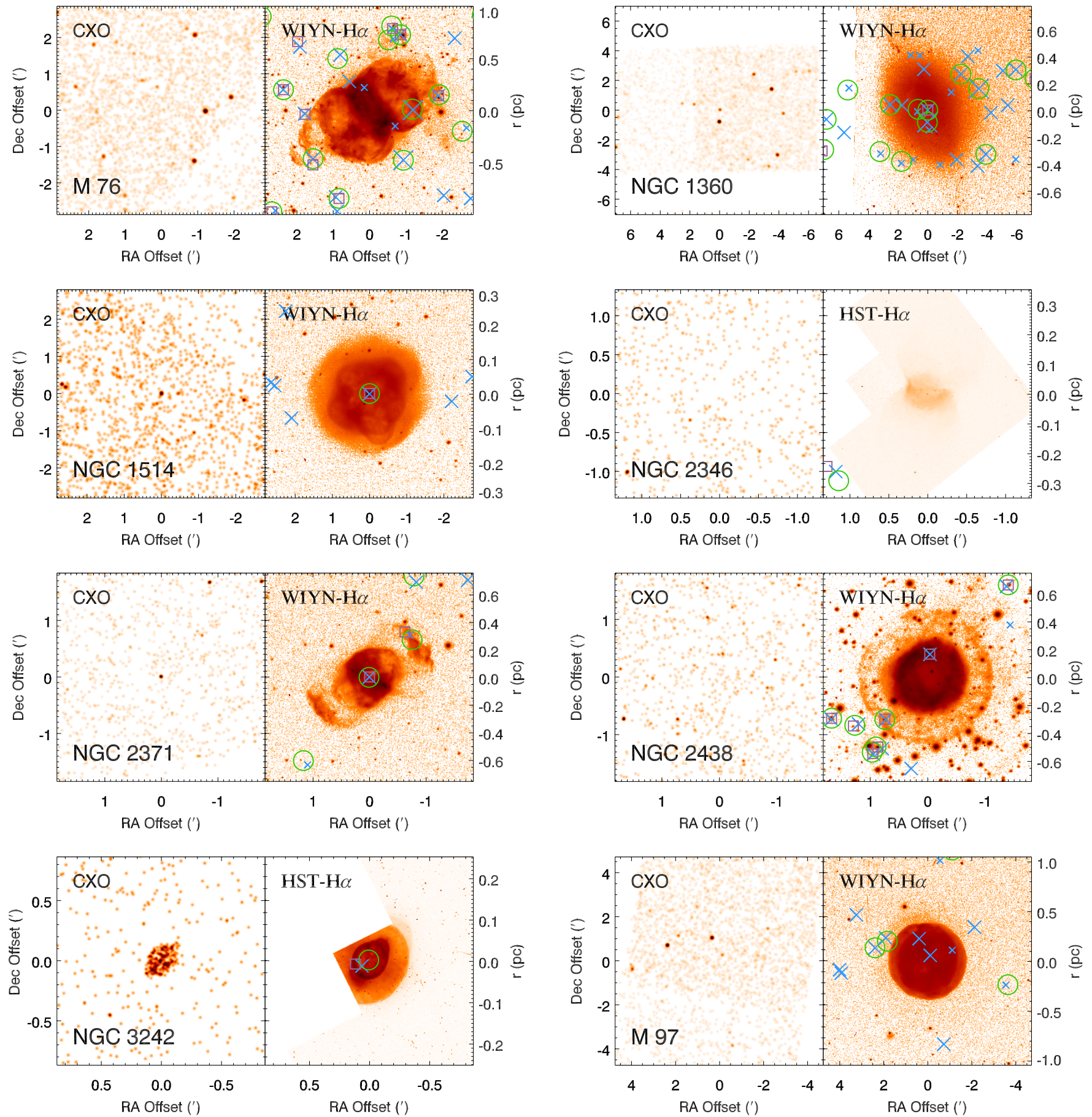

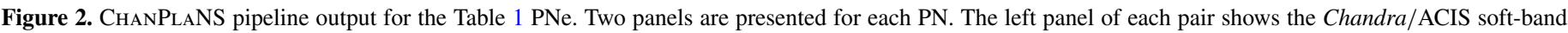

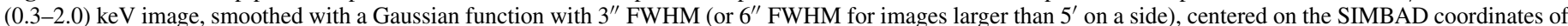

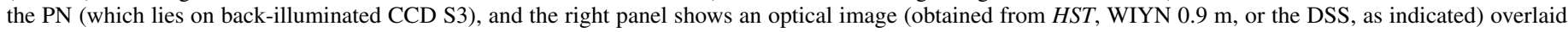

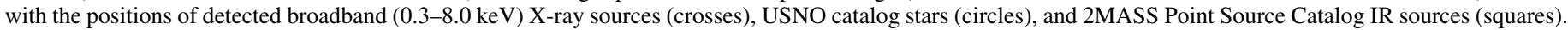
The size of the cross is proportional to the number of X-ray photons detected.

(A color version of this figure is available in the online journal.)

wavdetect (Freeman et al. 2002) with wavelength size scales of $1,2,4,8$, and 16 pixels. For sources within $4^{\prime}$ of the target PN, we use single pixel binning ( $0.492^{\prime \prime}$ pixels), while for sources at off-axis angles greater than $4^{\prime}$, we rebin the images $4 \times 4$ $\left(\sim 2^{\prime \prime}\right.$ pixels). The wavdetect source detection threshold is set such that the faintest sources identified and recorded are detected at the $\sim 3 \sigma$ significance level. The resulting lists of $\mathrm{X}$-ray sources are then cross-correlated with the USNO-B1 (Monet et al. 2003) and 2MASS ${ }^{26}$ (optical and near-infrared) Point Source Catalogs (PSCs), and the nearest optical and near-infrared sources within $10^{\prime \prime}$ of each detected X-ray source are identified and recorded. Thus far, identification of sources of extended (diffuse) X-ray

26 This publication makes use of data products from the Two Micron All Sky Survey, which is a joint project of the University of Massachusetts and the Infrared Processing and Analysis Center/California Institute of Technology, funded by the National Aeronautics and Space Administration and the National Science Foundation. 

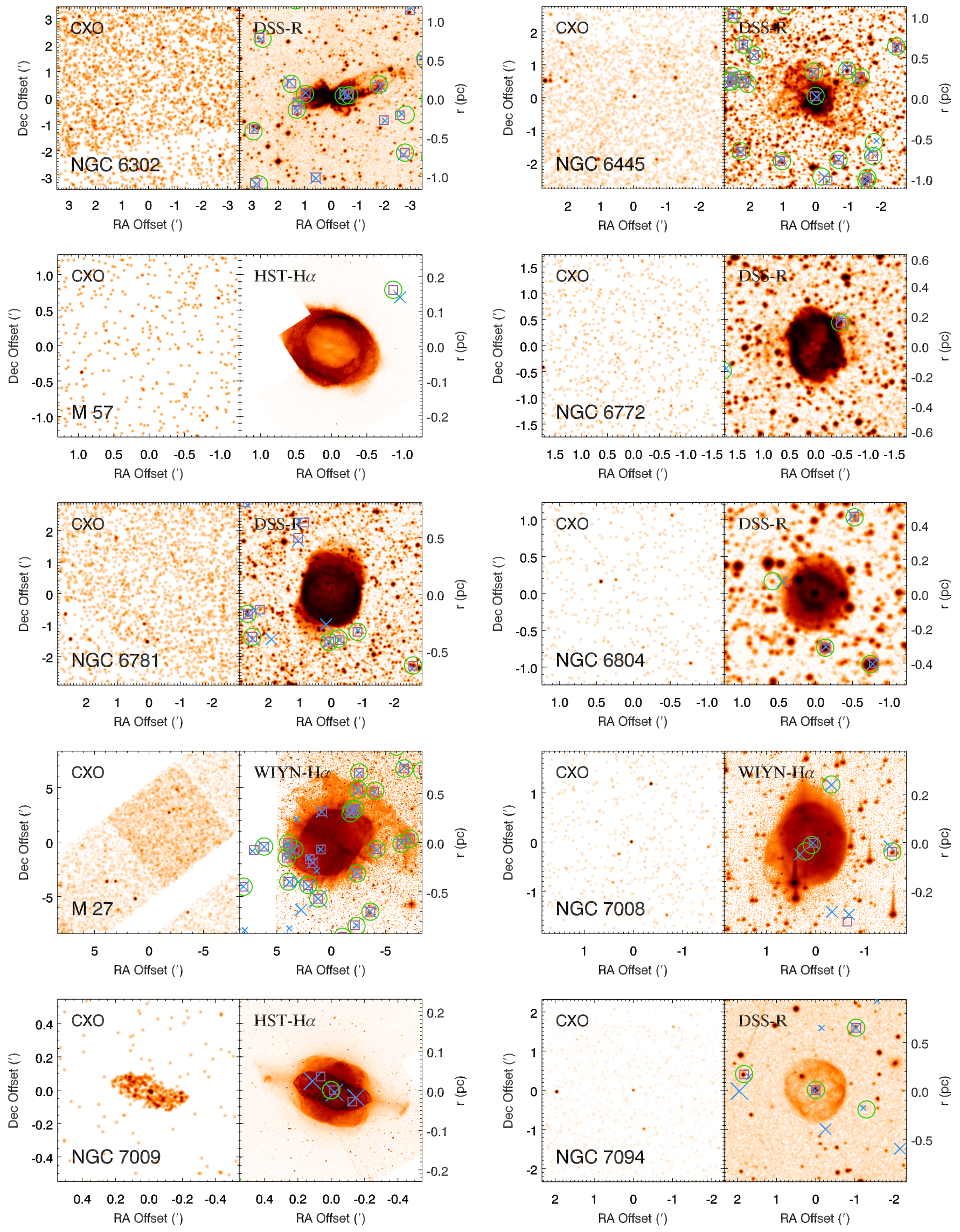

Figure 2. (Continued) 

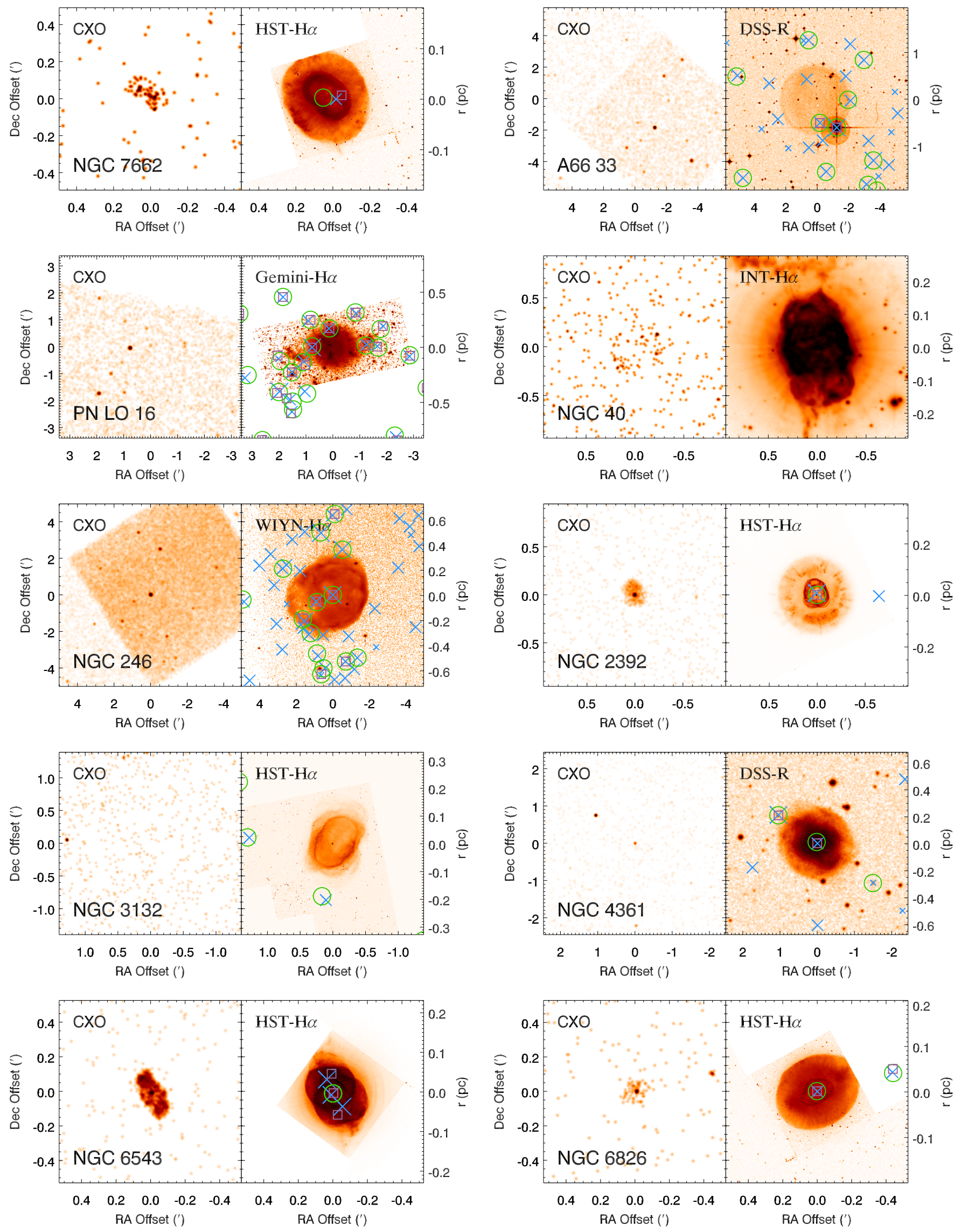

Figure 2. (Continued) 

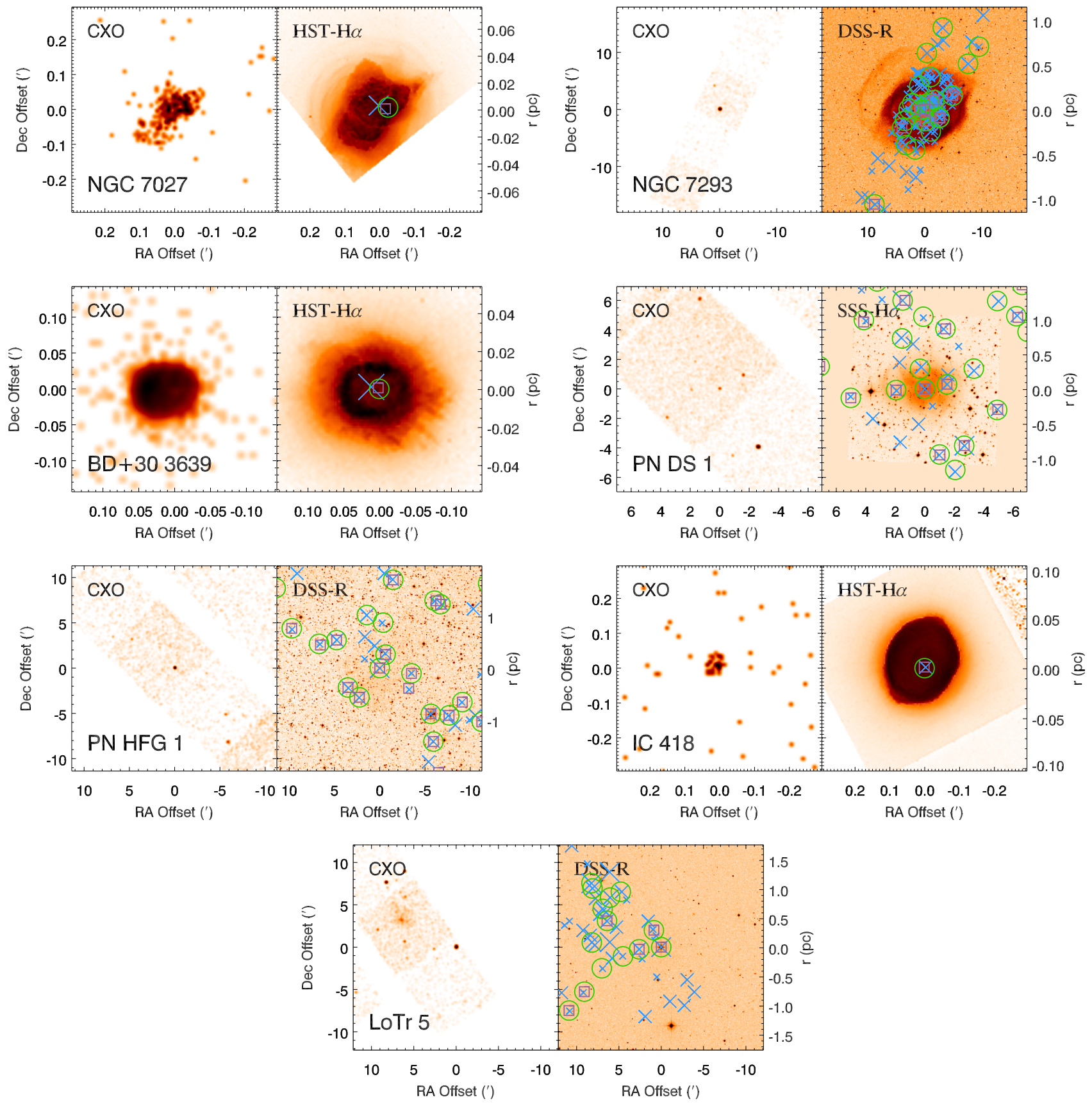

Figure 2. (Continued)

emission (Section 3.2) has been restricted to visual inspection of the soft-band (0.3-2.0 keV) images, assisted in some cases by image rebinning and/or smoothing.

\subsubsection{Source Event Statistics and Spectral Extraction}

For those PNe whose central stars are detected as X-ray sources (Sections 3.1 and 3.2), we calculate statistics for the events in a 3 ".5 radius region centered on the CSPN, so as to determine the total number of source photons and the mean, median, and first and second quartile photon energies. The median energy is an observed quantity that is dependent on instrumental energy response; for an ensemble of sources observed with a particular instrument, however, median photon energy is indicative of the source plasma temperature and intervening absorbing column (Getman et al. 2010). We also perform spectral extractions within regions of interest encompassing the
CSPN, nebula (both including the central star and excluding the central star), and source-free background regions. The sizes and morphologies of the nebular extraction regions are determined from the optical morphologies of the nebula. This extraction-which results in generation of source and background region X-ray spectra and all associated Chandra/ACIS response (source-specific calibration) files necessary for spectral model fitting - is performed for all objects, whether detected or not detected. Analysis of the resulting X-ray spectra will be presented in forthcoming CHAnPlaNS papers (e.g., R. Montez et al. 2012, in preparation).

\subsubsection{Pipeline Output: Annotated Images}

Figure 2 illustrates the results of the processing pipeline just described. The two panels included for each $\mathrm{PN}$-presented in the order listed in Table 1-display the Chandra soft-band 
Table 2

Log of Chandra Observations

\begin{tabular}{|c|c|c|c|}
\hline Name & OBSID & Date & $\begin{array}{c}\text { Exposure } \\
(\mathrm{ks})\end{array}$ \\
\hline A 33 & 12369 & 2011 Jan 21 & 29.67 \\
\hline $\mathrm{BD}+30$ & 587 & $2000 \operatorname{Mar} 21$ & 19.22 \\
\hline " & 10821 & 2009 Jan 22 & 38.63 \\
\hline " & 9932 & 2009 Jan 27 & 38.03 \\
\hline DS 1 & 9953 & 2009 Jul 19 & 24.15 \\
\hline HFG 1 & 9954 & 2008 Dec 11 & 11.45 \\
\hline IC 418 & 7440 & 2006 Dec 12 & 49.38 \\
\hline Lo 16 & 12367 & 2012 Jan 30 & 30.00 \\
\hline LoTr 5 & 3212 & 2002 Dec 4 & 27.74 \\
\hline NGC 40 & 4481 & 2004 Jun 13 & 19.89 \\
\hline NGC 246 & 2565 & 2002 Oct 22 & 40.95 \\
\hline NGC 650-51 & 12371 & 2010 Nov 15 & 29.90 \\
\hline NGC 1360 & 12362 & 2010 Nov 19 & 19.65 \\
\hline NGC 1514 & 12361 & 2010 Nov 4 & 20.78 \\
\hline NGC 2346 & 12379 & 2010 Dec 19 & 29.89 \\
\hline NGC 2371-72 & 12376 & 2012 Feb 18 & 29.67 \\
\hline NGC 2392 & 7421 & 2007 Sep 13 & 57.41 \\
\hline NGC 2438 & 3765 & 2003 Apr 21 & 49.75 \\
\hline " & 12377 & 2011 Feb 6 & 29.67 \\
\hline NGC 3132 & 4514 & 2004 Aug 8 & 23.97 \\
\hline NGC 3242 & 12380 & 2011 Feb 28 & 29.26 \\
\hline NGC 3587 & 12366 & 2011 Jul 5 & 19.24 \\
\hline NGC 4361 & 3760 & 2003 Feb 17 & 29.38 \\
\hline NGC 6302 & 14364 & 2012 Apr 25 & 7.47 \\
\hline "I & 12370 & 2011 Oct 25 & 22.54 \\
\hline NGC 6445 & 12375 & 2011 Feb 19 & 29.68 \\
\hline NGC 6543 & 630 & 2000 May 10 & 46.06 \\
\hline NGC 6543 & 11999 & 2009 Sep 26 & 25.03 \\
\hline NGC 6543 & 10443 & 2009 Sep 21 & 22.03 \\
\hline NGC 6720 & 12364 & 2011 Jan 23 & 19.80 \\
\hline NGC 6772 & 12372 & 2011 Jun 30 & 29.37 \\
\hline NGC 6781 & 12368 & 2011 Nov 22 & 28.25 \\
\hline NGC 6804 & 12378 & 2011 Nov 30 & 29.58 \\
\hline NGC 6826 & 8559 & 2007 Jul 24 & 15.04 \\
\hline " & 7439 & 2007 Jun 11 & 34.08 \\
\hline NGC 6853 & 12363 & 2010 Dec 17 & 19.80 \\
\hline NGC 7008 & 12365 & 2011 Nov 10 & 18.22 \\
\hline NGC 7009 & 12381 & 2011 Jun 25 & 29.66 \\
\hline NGC 7027 & 588 & 2000 Jun 1 & 18.22 \\
\hline NGC 7094 & 12374 & 2011 Apr 21 & 29.67 \\
\hline NGC 7293 & 631 & 1999 Nov 17 & 37.13 \\
\hline " & 1480 & 1999 Nov 18 & 11.02 \\
\hline NGC 7662 & 12373 & 2012 May 15 & 27.61 \\
\hline
\end{tabular}

$(0.3-2.0 \mathrm{keV}) \mathrm{X}$-ray image centered on the position of the PN (left panel) and the positions of Chandra-detected broadband (0.3-8.0 keV) X-ray sources, USNO-B1.0 catalog stars, and 2MASS PSC IR sources overlaid on an optical ( $\mathrm{H} \alpha$ or $R$ band) image (right panel). The Hubble Space Telescope (HST) optical $(\mathrm{H} \alpha)$ images in the right-hand panels were obtained from the Hubble Legacy Archive ${ }^{27}$ where available; otherwise, we display the ground-based images described in Section 2.2.2 or $R$-band images from the Digital Sky Survey ${ }^{28}$ (DSS).

\footnotetext{
27 Based on observations made with the NASA/ESA Hubble Space Telescope, and obtained from the Hubble Legacy Archive, which is a collaboration between the Space Telescope Science Institute (STScI/NASA), the Space Telescope European Coordinating Facility (ST-ECF/ESA) and the Canadian Astronomy Data Centre (CADC/NRC/CSA) where available; otherwise, http://archive.stsci.edu/hst

28 http://archive.stsci.edu/dss
}

Table 3

Planetary Nebula X-Ray Point Source Characteristics

\begin{tabular}{|c|c|c|c|c|}
\hline Name & $\begin{array}{c}N^{\mathrm{a}} \\
\text { (photons) }\end{array}$ & $\begin{array}{c}C^{\mathrm{b}} \\
\left(\mathrm{ks}^{-1}\right)\end{array}$ & $\begin{array}{c}\text { Median } E^{\mathrm{c}} \\
\quad(\mathrm{keV})\end{array}$ & $\begin{array}{c}E \text { Range }^{\mathrm{d}} \\
(\mathrm{keV})\end{array}$ \\
\hline $\mathrm{NGC} 40^{\mathrm{e}}$ & $\ldots$ & & $\ldots$ & \\
\hline NGC 246 & 749 & $18.5 \pm 0.3$ & 0.33 & $0.26-0.39$ \\
\hline NGC 650 & $\ldots$ & $<0.16$ & $\ldots$ & $\ldots$ \\
\hline NGC 1360 & 25 & $1.26 \pm 0.25$ & 0.27 & $0.17-0.56$ \\
\hline NGC 1514 & 13 & $0.62 \pm 0.17$ & 0.72 & $0.59-0.98$ \\
\hline NGC 2346 & $\ldots$ & $<0.13$ & $\ldots$ & $\ldots$ \\
\hline NGC 2371-72 & 29 & $0.96 \pm 0.18$ & 0.36 & $0.31-0.47$ \\
\hline NGC $2392^{\mathrm{e}}$ & 241: & 4.2: & 0.95: & $0.61-1.46$ \\
\hline NGC 2438 & $\ldots$ & $<0.13$ & $\ldots$ & $\ldots$ \\
\hline NGC 3132 & $\ldots$ & $<0.16$ & $\ldots$ & $\ldots$ \\
\hline $\mathrm{NGC} 3242^{\mathrm{e}}$ & $\ldots$ & $<0.5:$ & $\ldots$ & $\ldots$ \\
\hline NGC 3587 & $\ldots$ & $<0.25$ & $\ldots$ & $\ldots$ \\
\hline NGC 4361 & 43 & $1.48 \pm 0.22$ & 0.26 & $0.19-0.40$ \\
\hline NGC 6302 & $\ldots$ & $<0.17$ & $\ldots$ & $\ldots$ \\
\hline NGC 6445 & 33 & $1.10 \pm 0.19$ & 1.04 & $0.90-1.20$ \\
\hline $\mathrm{NGC} 6543^{\mathrm{e}}$ & 165: & 3.6: & $0.55:$ & $0.43-0.73$ \\
\hline NGC 6720 & $\ldots$ & $<0.20$ & $\ldots$ & $\ldots$ \\
\hline NGC 6772 & $\ldots$ & $<0.13$ & $\ldots$ & $\ldots$ \\
\hline NGC 6781 & $\ldots$ & $<0.13$ & $\ldots$ & $\ldots$ \\
\hline NGC 6804 & $\ldots$ & $<0.13$ & $\ldots$ & $\ldots$ \\
\hline NGC $6826^{\mathrm{e}}$ & $27:$ & 0.8: & 0.71: & $0.55-0.90$ \\
\hline NGC 6853 & 173 & $8.74 \pm 0.66$ & 0.18 & $0.17-0.20$ \\
\hline NGC 7008 & 23 & $1.26 \pm 0.26$ & 0.85 & $0.67-1.29$ \\
\hline NGC $7009^{e}$ & $31:$ & 1.0: & $0.74:$ & $0.57-1.01$ \\
\hline NGC $7027^{e}$ & $\ldots$ & $<0.5$ : & $\ldots$ & $\ldots$ \\
\hline NGC 7094 & 28 & $0.93 \pm 0.18$ & 0.55 & $0.41-0.95$ \\
\hline NGC 7293 & 396 & $35.9 \pm 1.8$ & 0.89 & $0.73-1.06$ \\
\hline NGC $7662^{\mathrm{e}}$ & $\ldots$ & $<0.5$ & $\ldots$ & $\ldots$ \\
\hline A 33 & $\ldots$ & $<0.13$ & $\cdots$ & $\cdots$ \\
\hline $\mathrm{BD}+30^{\circ} 3639^{\mathrm{e}}$ & $\ldots$ & $\ldots$ & $\ldots$ & $\ldots$ \\
\hline DS 1 & 55 & $2.29 \pm 0.31$ & 1.01 & $0.79-1.28$ \\
\hline HFG 1 & 143 & $12.6 \pm 1.0$ & 1.05 & $0.83-1.38$ \\
\hline IC $418^{\mathrm{e}}$ & $\ldots$ & $\ldots$ & $\ldots$ & $\ldots$ \\
\hline Lo 16 & 8 & $0.26 \pm 0.09$ & 1.09 & $0.92-1.84$ \\
\hline LoTr 5 & 285 & $10.27 \pm 0.61$ & 1.13 & $0.88-1.55$ \\
\hline
\end{tabular}

Notes.

a Number of source photons, after background subtraction.

b Source photon count rate.

${ }^{c}$ Median source photon energy.

d Source photon energy range (25th through 75 th percentiles).

e Point source counts, count rate (or upper limit), median energy, and energy ranges are uncertain due to presence of diffuse emission component.

\section{RESULTS}

Results from the Chandra observations listed in Table 2 and illustrated in Figure 2 are summarized in Tables 1 and 3. The rightmost column of Table 1 states whether or not the PN was detected and, in the case of a detection, whether the PN displays a point-like X-ray source at the CSPN, diffuse X-ray emission, or a combination of the two. Based on preliminary model fits to the spectra extracted for the X-ray-faintest objects in Table 2-i.e., the diffuse source and soft X-ray point sources within NGC 2371 (each of which displays a count rate $\sim 1 \mathrm{ks}^{-1}$ ) and the "hard X-ray" point source within Lo 16 (count rate $\sim 0.3 \mathrm{ks}^{-1}$ ) —we conservatively estimate that our sensitivity limits for diffuse and hard (soft) point-like X-ray sources are $\sim 10^{30}$ and $\sim 10^{29}$ $\left(\sim 10^{31}\right) \mathrm{erg} \mathrm{s}^{-1}$, respectively, at the limiting $(1.5 \mathrm{kpc})$ distance of the survey (where the soft source luminosity limit is strongly dependent on the intervening absorbing column along the line of sight to the CSPN). 
Table 4

Planetary Nebulae: Chandra X-Ray Detection Statistics

\begin{tabular}{lccc}
\hline \hline Category $^{\mathrm{a}}$ & $N^{\mathrm{b}}$ & $N_{P X}{ }^{\mathrm{c}}$ & $N_{D X}{ }^{\mathrm{d}}$ \\
\hline Entire sample & 35 & $18^{\mathrm{e}}(53 \%)$ & $11(31 \%)$ \\
\hline Round/elliptical, F08 & 28 & $16^{\mathrm{e}}(57 \%)$ & $10(36 \%)$ \\
Bipolar, F08 & 7 & $2(28 \%)$ & $1(14 \%)$ \\
\hline Round/elliptical/irregular, SMV11 & 20 & $11^{\mathrm{e}}(55 \%)$ & $6(30 \%)$ \\
Bipolar/multipolar, SMV11 & 15 & $7(47 \%)$ & $5(33 \%))$ \\
\hline Near-IR H ${ }_{2}$ not detected & 15 & $9(60 \%)$ & $9(60 \%)$ \\
Near-IR H ${ }_{2}$ detected & 13 & $3(24 \%)$ & $2(15 \%)$ \\
\hline Known binary CSPN & 13 & $9^{\mathrm{e}}(69 \%)$ & $1(8 \%)$ \\
\hline
\end{tabular}

Notes.

a Morphologies as listed and defined in Column 3 of Table 1 and associated footnotes; CSPN binary detections and $\mathrm{H}_{2}$ detections as listed, respectively, in Columns 9 and 10 of Table 1.

b Total number of sample PNe in each category.

c Number of PNe in each category displaying point-like X-ray emission in Chandra imaging.

${ }^{\mathrm{d}}$ Number of PNe in each category displaying diffuse X-ray emission in Chandra imaging.

e Includes tentative detection of Lo 16.

Table 3 lists basic characteristics of the point-like X-ray source (net background-subtracted photon counts, count rates, median energy, and energy ranges) - or point source nondetection (upper limit on count rate), as the case may be-that is associated with each PN. Comparisons of X-ray and optical emission morphologies for the Table 1 PNe detected as diffuse X-ray emission sources are presented in Figures 3 and 4. A summary of the PNe observed and detected, broken down into various object categories (i.e., primary PN morphology descriptor, as listed in Column 3 of Table 1 and described in associated footnotes, detection and nondetection of near-IR $\mathrm{H}_{2}$ emission, and known binary CSPNe), is presented in Table 4 and Figure 5. Discussions of individual diffuse and point-like PN $\mathrm{X}$-ray sources (including the implications of these sources for the origin and evolution of their "host" PNe), as well as presentations of the results of detailed modeling of individual sources, are deferred to subsequent papers (e.g., R. Montez et al. 2012, in preparation). Here, we present summaries of the main results.

\subsection{PNe Displaying Diffuse X-Ray Emission}

$B D+30^{\circ} 3639, I C 418, N G C 40,2371,2392,3242,6543$, 6826, 7009, 7027, and 7662: The Chandra observations reported here establish that these $11 \mathrm{PNe}$ are diffuse X-ray sources. The ChanPlanS and archival images of these PNe (as well as contours of these X-ray images overlaid on optical images) are displayed in Figure 3 and are grouped according to whether or not the PN also displays a point-like emission component at its central star.

BD+30³639 (Campbell's Star; Kastner et al. 2000; Yu et al. 2009) and NGC 6543 (Cat's Eye; Chu et al. 2001; Kastner et al. 2002) were the earliest established-and remain the best documented-examples of PNe displaying emission from wind-shock-generated "hot bubbles" (Figure 3). No X-rays appear to be specifically associated with the CSPN of $\mathrm{BD}+30^{\circ} 3639$. However, NGC 6543 represents a case study of a PN harboring both diffuse (hot bubble) and harder, pointlike (CSPN) X-ray emission components (Chu et al. 2001; Guerrero et al. 2001). Similarly, the archival CHANPLANS observations of NGC 2392 (Eskimo) and NGC 6826 and the Cycle 12 CHANPlaNS observation of NGC 7009 (Saturn) reveal that both soft diffuse and harder point-like (CSPN) emission components are also clearly present in these PNe (Figure 3; the diffuse and point-source X-ray components, respectively, of NGC 2392 and 6826 are the subject of forthcoming papers by N. Ruiz et al. 2012, in preparation; M. Guerrero et al. 2012, in preparation). The Chandra detection of relatively hard point source emission in NGC 2392 likely explains the energy dependence of the X-ray morphology apparent in the earlier XMM data; see Guerrero et al. (2005). Likewise, in the case of NGC 7009which was also previously detected by XMM (Figure 4, lower left; Guerrero et al. 2002)_Chandra's spatial resolution establishes the presence of an X-ray point source embedded within diffuse X-ray nebulosity (Figure 4, lower right). The remaining example of a PN harboring both diffuse and point-like X-ray emission, NGC 2371, displays a softer CSPN X-ray source and very faint diffuse emission that partially fills its central regions (Figure 3).

In an XMM observation (Figure 4, upper left), NGC 3242 (the Ghost of Jupiter nebula) is well detected and appears as a marginally extended, asymmetric X-ray source (Ruiz et al. 2011). However, as in the cases of NGC 2392 (Guerrero et al. 2005) and 7009 (Guerrero et al. 2002), the diameter of the inner nebula of NGC 3242 is similar to the width of the $X M M /$ EPIC (pn and MOS) point spread functions, rendering its $X M M \mathrm{X}$-ray morphology difficult to interpret and (in particular) the potential contribution from an X-ray-luminous CSPN impossible to ascertain. In CHANPLANS imaging (Figure 4, upper right), the X-ray emission from NGC 3242 is clearly established as diffuse, with a smooth surface brightness distribution that traces the inner shell, including the protrusions along the shell's major axis. Notably, no X-ray point source is evident at the CSPN of NGC 3242 in the CHANPLANS image. The morphologically similar NGC 7662 (the Blue Snowball nebula) is a new (Cycle 12 Chandra) X-ray detection. As in the case of NGC 3242, we detect only diffuse emission within NGC 7662.

All three low-excitation nebulae (as measured in terms of [O III] to $\mathrm{H} \beta$ line ratio) observed thus far by Chandra-BD $+30^{\circ} 3639$, IC 418 , and NGC 40 (all of which were observed prior to Cycle 12) - display diffuse X-rays from hot bubbles, and all three lack point source (CSPN) emission (Figure 3). Two of the three, BD+30 3639 and NGC 40, harbor late [WC]-type CSPNe with dense, fast winds, leading to the suggestion that WR-type central stars are efficient at generating X-ray-luminous wind-blown bubbles within PNe (Montez et al. 2005; Kastner et al. 2008). The CHANPlaNS detection of diffuse X-rays within NGC 2371, which harbors an early [WO]-type CSPN, further reinforces this notion. Indeed, the three objects with WR-type CSPNe in Table $1-\mathrm{BD}+30^{\circ} 3639$, NGC 40, and NGC 2371-appear to represent a sequence in which both CSPN effective temperature and $\mathrm{PN}$ radius increase as diffuse X-ray luminosity decreases; NGC 2371 is the only one of the three to display X-rays from its CSPN. While the CSPN of IC 418 has not been classified as a [WC] type, this CSPN is as cool as the late-type [WC] stars within BD+30 3639 and NGC 40 and, like such stars, it drives a relatively strong, fast wind (Cerruti-Sola \& Perinotto 1989).

In contrast to the PNe just discussed, all of which appear to display diffuse X-ray emission that is confined to"hot bubbles," NGC 7027 (which was observed early in the Chandra mission; Kastner et al. 2001, 2002) provides a rare, clear example of X-ray emission from collimated flows within a nearby PN (Figure 3; Kastner 2009). Specifically, the X-ray emission closely traces 

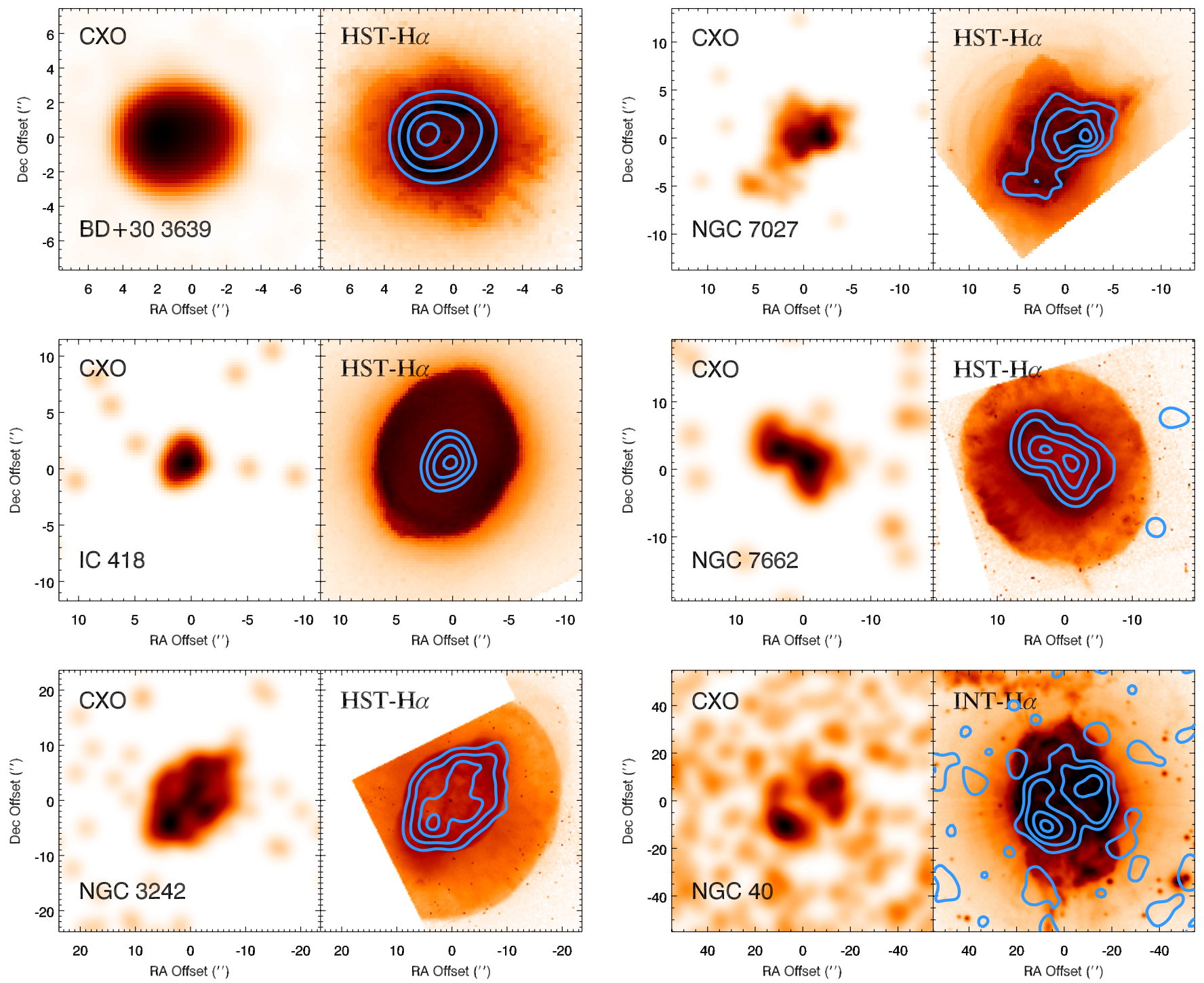

Figure 3. Images of Table $1 \mathrm{PNe}$ in which diffuse X-ray emission has been detected by Chandra. The left and right panel pairs for each PN display, respectively, Chandra $0.3-2.0 \mathrm{keV}$ images and Chandra contours overlaid on optical images. The Chandra images of all but two PNe (NGC 40 and NGC 2371) have been smoothed with a $3^{\prime \prime}$ FWHM Gaussian (the Chandra images of NGC 40 and NGC 2371 have been smoothed with a FWHM of 8"); contour levels are 10\%, 30\%, 60\%, and 90\% of the maximum X-ray surface brightness. The PNe in the first six panels display only diffuse emission; the PNe in the subsequent five panels display both diffuse and point-source X-ray emission components.

(A color version of this figure is available in the online journal.)

portions of the central, elliptical shell that have evidently been punctured by high-velocity bullets or jets (Cox et al. 2002).

\subsection{PNe Displaying Only Point-like X-Ray Emission at Central Stars}

In addition to the five PNe that display both diffuse and pointlike X-ray emission components, 13 objects display only pointlike X-ray emission from their CSPNe. These X-ray sources appear to belong to two general classes: very soft sources with median photon energies $<0.4 \mathrm{keV}$, most of which have hard X-ray "tails" (Section 3.2.1); and harder sources, with median photon energies ranging from $\sim 0.5 \mathrm{keV}$ to $\sim 1.0 \mathrm{keV}$ (Section 3.2.2).

\subsubsection{Objects with Strong or Dominant "Hot CSPN Photosphere" X-Ray Spectral Components}

$N G C$ 246, 1360, 4361, and 6853. These nebulae appear to represent a distinct group of CSPNe that display a combination of relatively high $T_{\text {eff }}$ and low median X-ray photon energy (Figure 6). However, only NGC 6853 (the Dumbbell nebula) displays an X-ray (ACIS-S3) SED that is consistent with "pure" photospheric emission from a hot ( $\gtrsim 100 \mathrm{kK})$ CSPN (this CHANPLANS result supports the previous analysis of ROSAT X-ray data by Chu et al. 1993). The detection of such emission from the CSPN of NGC 6583 by Chandra is facilitated by its proximity (see Table 1 and Section 3.3). As is evident in their detected photon energy ranges (Figure 6), each of the other CSPNe X-ray sources in this group-although significantly softer than the sources discussed in Section 3.2.2-displays a $\sim 0.4-0.6 \mathrm{keV}$ "tail" in its X-ray SED, indicative of excess emission above that expected from a hot CSPN photosphere (R. Montez et al. 2012, in preparation). The CSPN X-ray source within NGC 2371 (which shows very faint diffuse emission; Section 3.1.1) is also in this category, i.e., a "soft" source with a "hard tail" (Figure 6). The (archival) ACIS-S3 data for NGC 246 were previously published by Hoogerwerf et al. (2007), who found that the soft portion of the CSPN X-ray spectrum was 

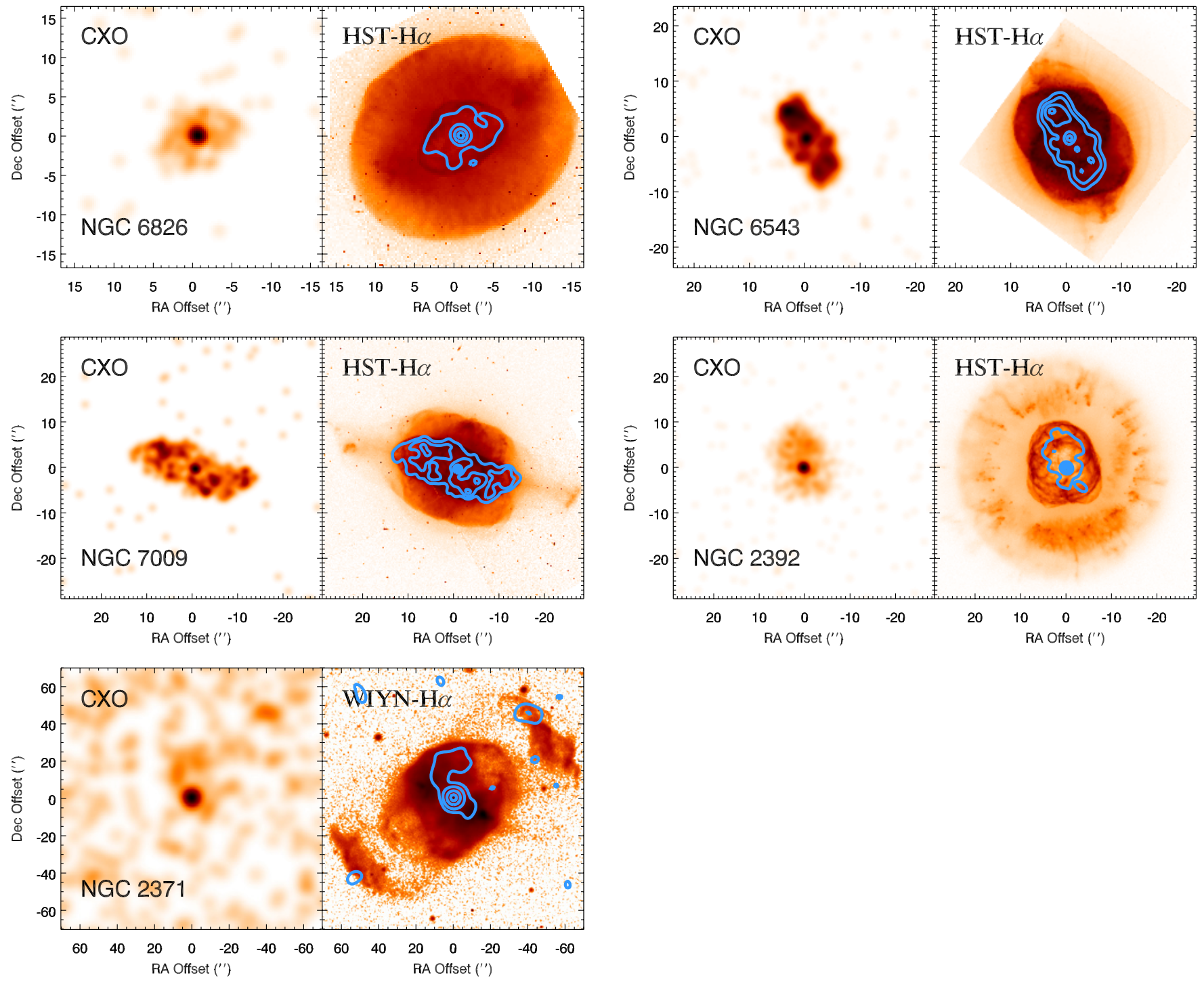

Figure 3. (Continued)

consistent with non-LTE models describing PG1159 star atmospheres, but that an additional component (consisting of emission lines of highly ionized C) was necessary to account for excess flux in the $0.3-0.4 \mathrm{keV}$ energy range.

\subsubsection{Objects with Strong or Dominant "Hard" $X$-Ray Spectral Components}

NGC 1514, 6445, 7008, 7094, 7293, DS 1, HFG 1, Lo 16, LoTr 5. NGC 7293 (Helix) is the prototype of an X-rayluminous CSPN whose X-ray SED extends to energies far too high to be explained as due to a hot pre-WD photosphere (Guerrero et al. 2000, 2001). Cycle 12 ChanPlaNS and archival observations have yielded a dozen more examples of such hard $\mathrm{X}$-ray excesses at CSPNe (Figure 6), including point sources clearly associated with five PNe that display "hot bubble" X-ray emission (Section 3.1). All of these CSPN X-ray sources have median X-ray energies in the range $\sim 0.5-1.0 \mathrm{keV}$, i.e., a factor $\sim 2-5$ larger than the CSPN X-ray sources described in Section 3.2.1. Remarks on most of these "hard X-ray CSPNe" follow.

NGC 1514. This PN has an unremarkable, amorphous optical morphology, but mid- to far-infrared imaging has revealed striking bipolar, double-ring dust structures exterior to the ionized nebula (Ressler et al. 2010; Aryal et al. 2010). The central star has a companion of type A0 (Ciardullo et al. 1999) — the earliest spectral type among known binary companions of "hard X-ray CSPNe" (see Section 4.2).

NGC 6445. This object is a bipolar PN with faint lobes and a bright central ring or torus. The central, point-like X-ray source detected in CHANPLANS imaging, which is offset by $\sim 3^{\prime \prime}$ from the SIMBAD coordinates of the PN, may be the first secure detection of the central star (or central binary, as the case may be) of this PN. This X-ray source is slightly off-center within the central ring.

NGC 7008. This PN displays an X-ray point source coincident with the position of its central star as listed in the HST Guide Star Catalog and 2MASS PSC. The central star has a probable late-type $(\mathrm{G})$ companion at separation $\sim 160 \mathrm{AU}$ (Ciardullo et al. 1999).

NGC 7293. This PN displays a composite $X$-ray SED consisting of a soft "hot blackbody" component and harder, highertemperature component (Guerrero et al. 2001). As in the cases of known binary CSPNe (see next), the harder $\mathrm{X}$-ray component may arise in the corona of a late-type 

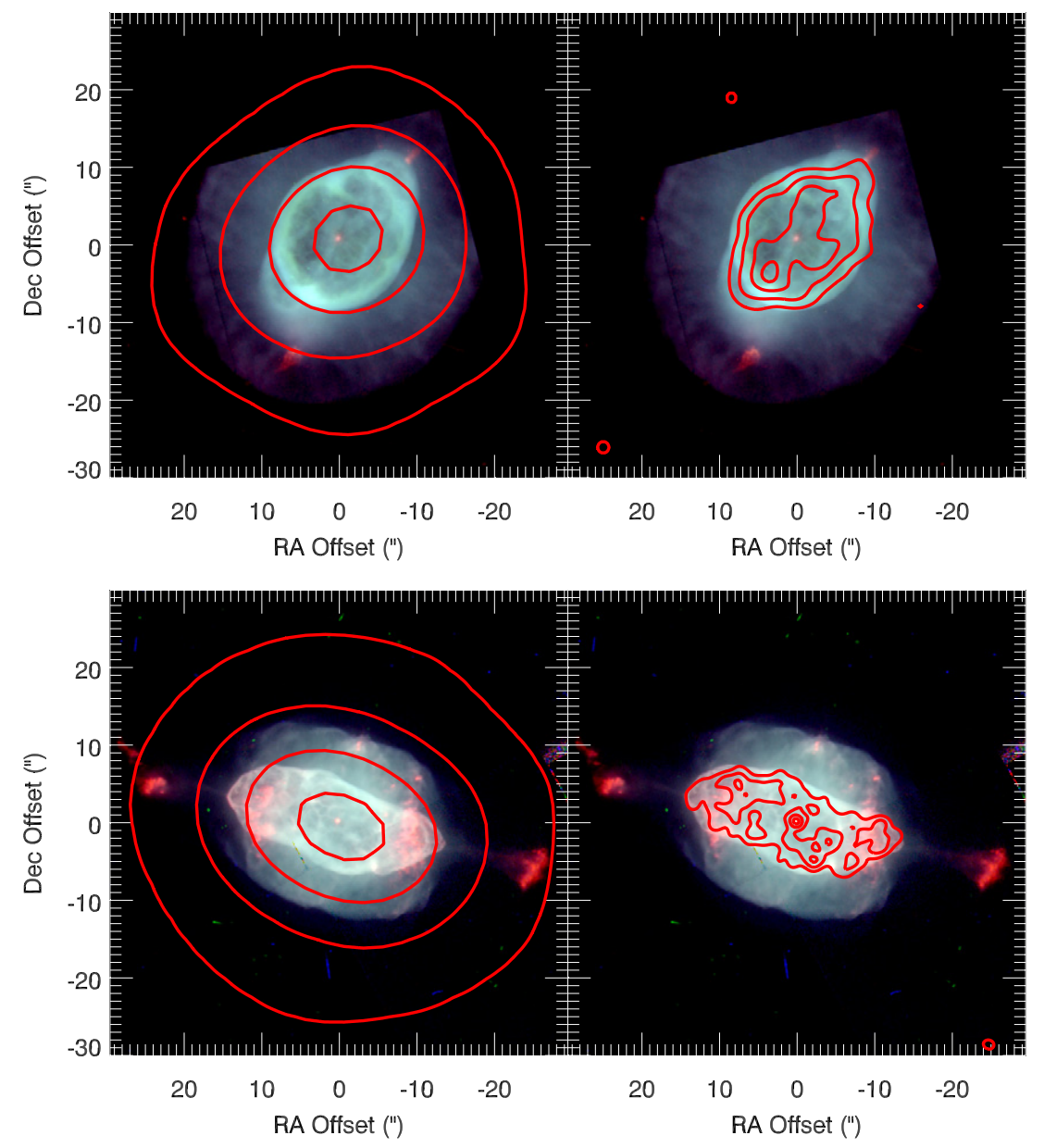

Figure 4. Color montages of HST archival images of NGC 3242 (top panels) and NGC 7009 (bottom panels) overlaid with contours of X-ray surface brightness as imaged by XMM (left panels) and Chandra (right panels). Contour levels are $10 \%, 30 \%, 60 \%$, and $90 \%$ of the peak.

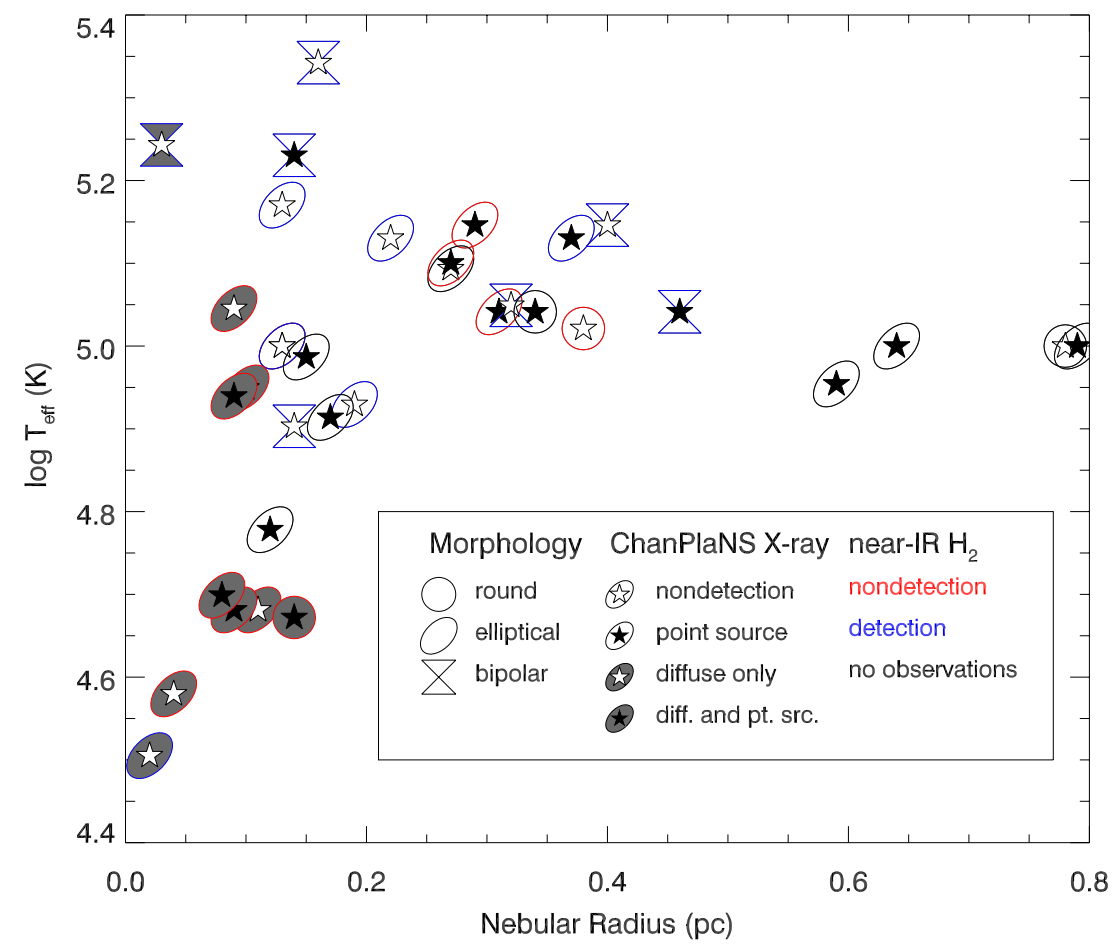

Figure 5. Plot of CSPN $T_{\text {eff }}$ vs. PN radius for the Table 1 objects, with symbols indicating presence or absence of diffuse or point-like X-ray emission, as well as PN morphology and presence or absence of $\mathrm{H}_{2}$ emission (see Table 1 and associated footnotes, and Table 4). 


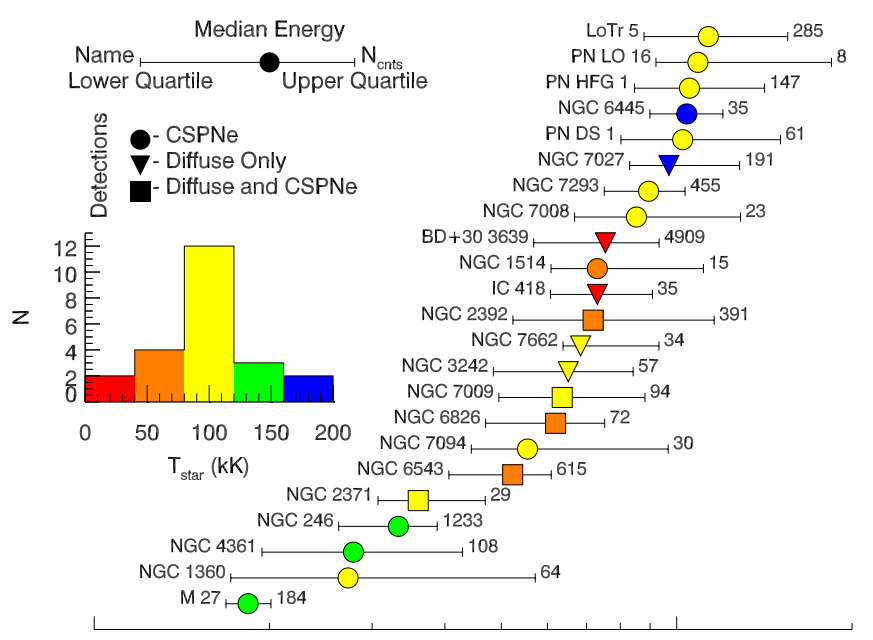

0.1

Energy (keV)

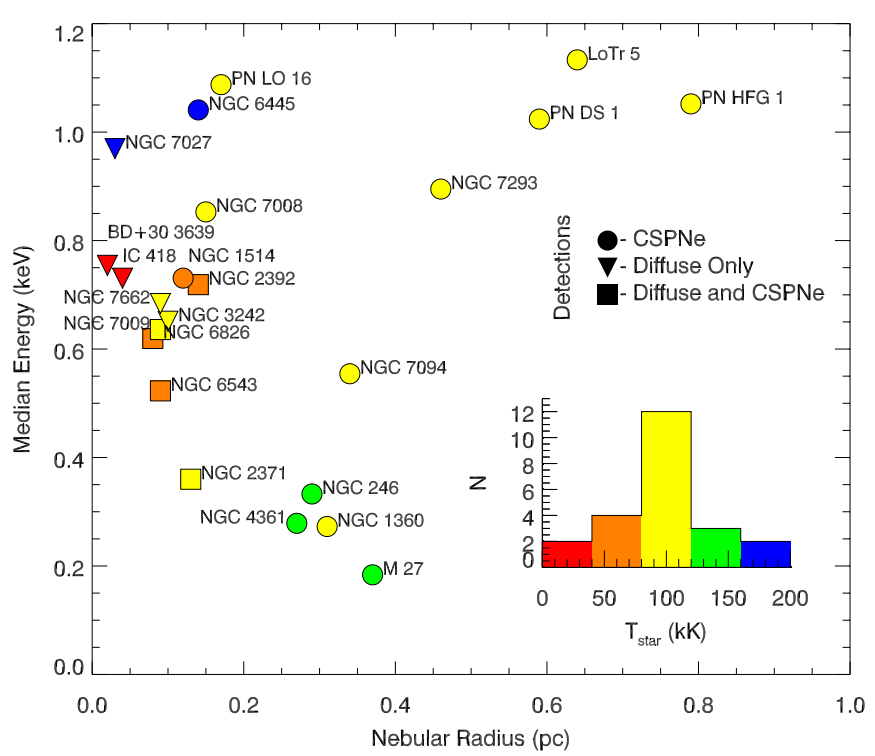

Figure 6. Top: photon energy statistics (net source counts, median energy, and first and third quartile energies) for PN X-ray sources, ordered from lowest to highest median energy (bottom to top). Symbols indicate nature of emission (point-like at CSPN, diffuse, or both CSPN and diffuse) and are color-coded according to CSPN $T_{\text {eff }}$ (see inset histogram, which also displays the distribution of $T_{\text {eff }}$ for CSPNe detected as X-ray sources). Bottom: PN X-ray source median energy vs. PN radius, with symbols as in the top panel.

(dM) companion - a possibility bolstered by detection of variable $\mathrm{H} \alpha$ emission from the CSPN (Gruendl et al. 2001). Additional supporting evidence for the presence of such a companion remains elusive, however (e.g., O’Dwyer et al. 2003).

DS 1, HFG 1, and LoTr 5. All three PNe feature late-type companions to their CSPNe. The PNe DS 1 and HFG 1 were targeted by Chandra because their CSPNe are known close binaries and, hence, candidate post-common envelope objects, while the companion to the CSPN of LoTr 5, which was observed serendipitously, is a Ba-rich giant (Montez et al. 2010 and references therein). In each case, the X-ray characteristics of the central, point-like source are consistent with coronal emission-as expected if the source is a late-type binary companion that has been spun up by accretion of material lost by the PN progenitor (see Section 4.2 and Montez et al. 2010).
Lo 16. This chaotic nebula that also harbors a (close, 0.49 day period) binary CSPN (D. Frew et al. 2012, in preparation) and is very tentatively detected in Cycle 12 imaging as a rather hard CSPN X-ray source (median energy $\sim 1.1 \mathrm{keV}$ ).

\subsection{X-Ray Nondetections}

NGC 650-1, 2346, 2438, 3132, 3587, 6720, 6772, 6781, 6804, A33. The majority of these X-ray-nondetected objects are molecule-rich PNe (Kastner et al. 1996) with morphologies that are either sharply bipolar (NGC 650-1, 2346) or Ringlike (NGC 3132, 6720, 6772, 6781). The Ring-like PNe likely have intrinsically axisymmetric density structure-fundamentally similar to the structures of clearly morphologically bipolar (pinched-waist) PNe such as NGC 650-1 and NGC 2346 (Kastner et al. 1994). NGC 2346 also was undetected in XMM imaging (Gruendl et al. 2006), but the Cycle 12 CHanPlanS nondetection places more severe constraints on the X-ray luminosity of its CSPN (R. Montez et al. 2012, in preparation). Tarafdar \& Apparao (1988) reported a $\sim 3 \sigma$ Einstein X-ray Observatory detection of A33, but CHANPLANS imaging demonstrates this association is spurious, and the Einstein source can most likely be attributed to an X-ray-luminous (and optically bright) field star near the southwest edge of the nebula, possibly combined with 1-2 weaker field X-ray sources within the boundaries of the PN. Although the CSPN of NGC 3587 was detected as a very soft X-ray source by ROSAT (Chu et al. 1998), its nondetection here is not surprising, given its lower luminosity, lower photospheric temperature, and larger distance relative to the CSPN of NGC 6583 (Section 3.2.1).

\section{DISCUSSION}

The sample of $35 \mathrm{PNe}$ within $\sim 1.5 \mathrm{kpc}$ observed to date by Chandra affords the first opportunity for relatively unbiased statistical investigations of the spatial and spectral characteristics of PN X-ray emission, so as to inform studies of PN formation and evolution. The initial sample (Table 1) is still rather heterogeneous and prone to selection effects, as it is composed of a mixture of high-excitation PNe and small subsets of objects specifically targeted for various reasons (Section 2.1); furthermore, data analysis is still in its early stages. Hence, it is premature to draw firm conclusions concerning the nature(s) of X-ray sources within PNe, much less the implications of PN $\mathrm{X}$-ray emission (or lack thereof) for the origin and shaping of PNe. Nevertheless, a few preliminary trends are apparent in the initial CHANPLANS results described in Section 3. We highlight and comment on these trends in the following subsections.

\subsection{Diffuse X-Ray Emission from $P N e$}

Ten of the eleven sample PNe displaying diffuse emission (Section 3.1; Figure 3) are classified by Frew (2008) as elliptical or round nebulae (the lone exception being NGC 7027, which is classified as bipolar by Frew 2008). These diffuse X-ray PNe are also generally molecule-poor (e.g., they lack detections of near-IR $\mathrm{H}_{2}$ emission; see Table 4 and Kastner et al. 1996), with the notable exceptions of BD $+30^{\circ} 3639$ and NGC 7027. In optical imaging, the diffuse X-ray PNe display multiple, nested shells with well-defined innermost bubbles (Frew morphology subclass of " $m$ " and/or Sahai et al. secondary morphology characteristic of "i" in Table 1) and, in all but one PN, the diffuse X-ray emission lies within the confines of these elliptical inner bubbles (the lone exception is, once again, NGC 7027; 
Kastner et al. 2001, 2002). Most of the "hot bubble X-ray" PNe (Section 3.1) also show ansae (Sahai et al. secondary morphology characteristic of "a" in Table 1) associated with bullet-like mass ejections (FLIERS; Balick et al. 1994), and four objects (NGC 40, 2371, 6543, 7009) display axisymmetric and/or point-symmetric structures that are further indicative of fast, collimated flows, leading to classifications of bipolar, multipolar, or "collimated lobe pair" nebulae under the PN classification system of Sahai et al. (2011).

Figure 5 and Table 1 readily demonstrate that (1) the central bubbles within all of the diffuse X-ray PNe have radii $\lesssim 0.15 \mathrm{pc}$, corresponding to dynamical ages $\lesssim 5 \times 10^{3}$ yr; and (2) most diffuse X-ray PNe have inferred CSPN effective temperatures $T_{\text {eff }} \lesssim 100 \mathrm{kK}$ (the only exceptions thus far being NGC 7027 and 7662). These two observations suggest, respectively, that (1) the timescale for energetic wind interactions in elliptical $\mathrm{PNe}$ is $\sim 5 \times 10^{3} \mathrm{yr}$; and (2) the luminous X-ray emission arising in wind shocks may contribute to the high-excitation states of the subclass of multiple-shell elliptical PNe that display welldefined central bubbles (provided such nebulae harbor sufficient masses of high-density gas; Ercolano 2009).

The foregoing results reinforce previous assertions by Gruendl et al. (2006), Kastner et al. (2008), and Kastner (2009) that the necessary conditions for detectable diffuse X-ray emission in PNe are either (1) a combination of energetic central star (pre-WD) winds and enclosed inner PN shells (or lobes) that can effectively confine wind-shock-heated plasma, as is the case for all CHANPLANS survey objects apart from NGC 7027; or (2) high-velocity, collimated post-AGB flows impinging on AGB ejecta, as is the case for NGC 7027 (as well as for the more distant objects Mz 3 and Hen 3-1475; Kastner et al. 2003; Sahai et al. 2003). Moreover, in the former case, it appears that the conditions described in the interacting winds scenarios which predict the production of a classical "hot bubble" (e.g., Zhekov \& Perinotto 1996) are met only for a limited class of PNe: specifically, elliptical, nested-shell PNe with "young" inner bubbles and ansae. This result appears to underscore the importance of accounting for the rapid time evolution of pre-PN and CSPN wind properties in modeling the key, early stages in the structural evolution of PNe (see, e.g., Villaver et al. 2002; Akashi et al. 2006; Huarte-Espinosa et al. 2012). Additional analysis of the CHANPLANS and archival data obtained to date, combined with further Chandra X-ray observations of PNe, should lead to an improved understanding of the potential implications of PN diffuse X-rays for models of the evolution of CSPN temperatures, masses, and winds, as well as the consequences of such CSPN evolution on the surrounding PN.

\subsection{Point-like X-Ray Emission from CSPNe}

It is apparent from Figure 5 and Table 4 that, like diffuse X-ray emission, point-like (CSPN) X-ray emission is more often associated with molecule-poor than molecule-rich $\left(\mathrm{H}_{2}\right.$-detected) $\mathrm{PNe}$; furthermore, the majority of $\mathrm{PNe}$ with X-ray-luminous central stars have elliptical or round morphologies, according to the Frew (2008) classifications. Specifically, $~ 60 \%-65 \%$ of elliptical (or round), molecule-poor PNe harbor CSPN X-ray sources, whereas only $\sim 25 \%$ of PNe with bipolar morphologies and/or in which near-IR $\mathrm{H}_{2}$ has been detected display such CSPN X-ray point sources. It also appears that the majority of PNe hosting X-ray-luminous central stars have morphologies that are perhaps best characterized as amorphous and internally disorganized-in stark contrast to the (generally) highly structured morphologies of PNe that display diffuse X-ray emission from hot bubbles (Sections 3.1.1 and 4.1) or that lack detectable X-rays (Section 3.3).

The X-ray SEDs of these CSPN X-ray sources appear to represent two general classes (Figure 6, top): (1) objects that display very soft X-ray SEDs, indicative of strong or dominant hot ( 100-200 kK) photospheric components (Section 3.2.1), and (2) CSPNe that display harder X-ray SEDs, dominated by photons in the range $\sim 0.6-1.0 \mathrm{keV}$ (Section 3.2.2). The former (soft X-ray) CSPN group, all of which have rather high CSPNe $T_{\text {eff }}$, are thus far confined to a rather narrow range in $\mathrm{PN}$ radius (i.e., radii $\sim 0.1-0.4 \mathrm{pc}$; Figure 6 , bottom), corresponding to a short, well-defined dynamical timespan. This suggests that the epoch of significant X-ray contributions from hot CSPN photospheric radiation is both delayed and short-lived. Specifically-setting aside the young, [WO]-type CSPN in NGC 2371-it appears that the epoch of detectable soft X-ray emission from the CSPN photosphere corresponds to a dynamical PN age of $\sim 10^{4}$ yr (Table 1), and that such photospheric X-ray emission then declines significantly after another few $\times 10^{3} \mathrm{yr}$.

The second (harder X-ray SED) group represents the majority of CSPN X-ray sources. Unlike the soft CSPN point sources, the harder CSPN X-ray sources span a wide range of PN radii, indicating that either a single long-lived process or a combination of short-lived and longer-timescale processes, intrinsic to the PN stellar component, are responsible. There is a broad range of potential explanations for these CSPN sources, including (see Guerrero et al. 2001; Blackman et al. 2001a; Soker \& Kastner 2002; Montez et al. 2010; Bilíková et al. 2010, and references therein): coronal emission from late-type binary companions that have been "spun up" (and hence become highly magnetically active) via accretion of mass lost by the PN progenitor, or whose coronae have been compressed by the CSPN wind; post-AGB magnetic activity at the CSPN itself (possibly instigated by interactions with a past or present binary companion); emission arising from an actively accreting companion (e.g., accretion shocks at a mainsequence companion, or an accretion disk associated with a compact companion); re-accretion ("fallback") of PN material onto the CSPN; or self-shocking, variable, fast CSPN winds analogous to those of massive OB stars. The data available thus far do not particularly favor any one of these alternative models; indeed, it is likely that different mechanisms may apply to different CSPNe. However, there are several points worth noting.

1. The three X-ray CSPNe for which the most likely cause of the X-rays is coronal emission from spun-up companions, DS 1, HFG 1, and LoTr 5 (Montez et al. 2010), are associated with some of the dynamically oldest (largest) PNe in the ChanPlaNS sample (Figure 6, bottom). This is consistent with the notion that the spin-down (hence enhanced magnetic activity) timescale for the companions in these systems should be significantly greater than characteristic PN lifetimes $\left(\sim 10^{5}\right.$ yr; e.g., Frew 2008 and references therein).

2. The median energies of the DS 1, HFG 1 , and LoTr 5 CSPN $\mathrm{X}$-ray sources are very similar, and are among the hardest thus far detected $(\sim 1.0 \mathrm{keV})$, reflecting the relatively high temperatures of these sources $\left(T_{X} \sim 10 \mathrm{MK}\right.$, consistent with coronal emission from late-type companions; Montez et al. 2010). This suggests that other X-ray sources in this same median energy range (i.e., those within NGC 6445 and 7008; Figure 6) may also be due to the coronae of spun-up, 
late-type CSPN companions-an assertion bolstered by the disproportionately large fraction of PNe with known binary CSPN that display point-like X-ray sources (Table 4). On the other hand, in the case of the point source within NGC 7293-whose median X-ray photon energy is similar to the other PNe CSPN X-ray sources just mentioned-the presence of a CSPN companion earlier than late $\mathrm{M}$ spectral type is excluded (O'Dwyer et al. 2003).

3 . The constraints placed on the intrinsic X-ray luminosities of the CSPNe within NGC 650-1 and 2346 by their Chandra nondetections are compromised somewhat by the fact that these CSPNe suffer considerable extinction within the equatorial regions of their host bipolar PNe. Such absorption effects are important for the class of very soft (blackbody-dominated) CSPN sources (Section 3.2.1). On the other hand, our detection of the CSPN of the bipolar PN NGC 6445 indicates absorption-related selection effects are less important for sources with median energies $\gtrsim 0.6 \mathrm{keV}$ (S 3.2.2). Furthermore, the X-ray-undetected CSPNe of the similarly molecule-rich, "Ring-like" objects NGC 3132, 6720, 6772, 6781-which are lower-inclination analogs to bipolar PNe (Kastner et al. 1994, 1996)—are subject to relatively small line-of-sight absorbing columns. Hence, the initial CHANPLANS results suggest that strongly axisymmetric, molecule-rich $\mathrm{PNe}$ generally have X-rayfaint CSPNe.

4. The presence of a binary companion to the CSPN is widely believed to be responsible for bipolar (axisymmetric) structure in PNe (see, e.g., Balick \& Frank 2002 and references therein). The apparent lack of X-ray point sources associated with the central stars of molecule-rich, axisymmetric (bipolar and Ring-like) PNe among the CHANPLANS sample (Table 4) would therefore appear to contradict the hypothesis that spun-up companions are widely responsible for "hard X-ray CSPNe." It is possible that the lack of X-rayluminous CSPNe within bipolar and Ring-like PNe reflects the fact that these objects are descended from progenitors whose masses are higher than average for PNe $\left(\gtrsim 1.5 M_{\odot}\right.$; Kastner et al. 1996, and references therein); hence, on average, the central stars of bipolar and Ring-like PNe may have a higher incidence of X-ray-inactive, intermediate-mass binary companions than elliptical and round PNe. Indeed, the nondetection of X-ray sources at the CSPNe of NGC 2346 and 3132 is not inconsistent with these CSPNe harboring wide-separation, intermediate-mass companions; such (A type) stars are generally less magnetically active than late-type stars, due to their lack of envelope convective zones (although it is then noteworthy that the CSPN of NGC 1514, which has an A-type companion, is an X-ray source). Regardless, the relative rarity of CSPN X-ray emission in the case of other bipolar and Ring-like PNe is difficult to explain, if a close companion was responsible for their axisymmetric structures. Perhaps the companion is a magnetically inactive, nonaccreting WD, or has already merged with the CSPN (during, e.g., a common envelope phase). Alternatively, the lack of point-like X-ray emission from CSPNe of bipolar and Ring-like PNe may somehow reflect the more rapid evolution of such objects.

5. The X-ray point sources associated with "hot bubble X-ray" PNe (Section 3.1) display a narrow range of median photon energy ( $\sim 0.5-0.7 \mathrm{keV}$; Figure 6 , bottom). Though it remains to assess to what extent these median energies are affected by contamination from the underlying diffuse
X-ray emission, their clustering in median energy may suggest a common CSPN X-ray emission mechanism. This mechanism could be internal (small-scale) wind shocks in the near-CSPN environment, given that these PNe all exhibit the effects of ongoing wind collisions at large scales.

\section{SUMMARY}

We are undertaking CHANPlaNS, the first systematic Chandra X-Ray Observatory survey of PNe in the solar neighborhood. CHanPlaNS began with a 570 ks Chandra Cycle 12 Large Program targeting 21 (mostly high-excitation) PNe within $\sim 1.5 \mathrm{kpc}$ of Earth. We have combined the results of these observations with those obtained from Chandra archival data for the (14) other PNe within $\sim 1.5 \mathrm{kpc}$ that have been observed to date. The highlights of the early CHANPLANS results include the following.

1. The overall X-ray detection rate for the $35 \mathrm{PNe}$ within $\sim 1.5 \mathrm{kpc}$ observed thus far by Chandra is $\sim 70 \%$.

2. Roughly $50 \%$ of the sample PNe harbor X-ray-luminous point sources at their CSPNe. This fraction includes nine new detections of CSPNe X-ray sources among the Cycle 12 sample $\mathrm{PNe}$, and another three CSPN point sources identified via analysis of previously unpublished archival data.

3. All but one of the point-like X-ray sources detected at CSPNe display X-ray spectra that are harder than expected from hot cores emitting as simple blackbodies (the lone apparent exception is the central star of the Dumbbell nebula, NGC 6853). These hard X-ray excesses may suggest a high frequency of binary companions to CSPNe. Other potential explanations include self-shocking winds or PN mass fallback.

4. Soft, diffuse X-ray emission tracing shocks (in most cases, "hot bubbles") formed by energetic wind collisions is detected in $\sim 30 \%$ of the sample PNe. The PNe detected as diffuse X-ray sources include four nebulae imaged by Chandra in Cycle 12 (NGC 2371, 3242, 7009, 7662) and three PNe for which archival X-ray images are presented here for the first time (NGC 2392, 6826; IC 418).

5. Five objects (NGC 2371, 2392, 6543, 6826, and 7009) display both diffuse and point-like emission components in Chandra imaging.

6. The presence (or absence) of X-ray sources appears correlated with PN density structure: molecule-poor, elliptical nebulae are more likely to display X-ray emission (either point-like or diffuse) than molecule-rich, bipolar, or Ringlike nebulae.

7. In addition to displaying elliptical morphologies, most $\mathrm{PNe}$ detected as diffuse X-ray sources have a nested shell/halo structure and display bright ansae; the diffuse $\mathrm{X}$-ray emission regions are enclosed within the innermost, compact, sharp-rimmed shells. All of these inner shells have dynamical ages $\lesssim 5 \times 10^{3} \mathrm{yr}$, placing firm constraints on the timescale for strong shocks due to wind interactions in PNe.

8. The central stars of all but two diffuse X-ray-emitting PN-the exceptions being NGC 7027 and 7662-have effective temperatures $T_{\text {eff }} \lesssim 100 \mathrm{kK}$, further reflecting the youth of these objects and suggesting that high-energy emission arising in wind shocks may contribute to the highexcitation states of archetypical "hot bubble" nebulae such as NGC 2392, 3242, 6826, and 7009. 
Further analysis of these and future CHANPLANS X-ray imaging spectroscopy data and results describing both point-like and diffuse X-ray emission from PNe will serve to inform and refine models describing PN shaping mechanisms and, in particular, the role of binarity in determining PN structure and evolution.

This research was supported via award number GO1-12025A to RIT issued by the Chandra X-ray Observatory Center, which is operated by the Smithsonian Astrophysical Observatory for and on behalf of NASA under contract NAS803060. M.A.G. acknowledges partial support by grant AYA2011-29754-C0302 of the Spanish MEC (co-funded by FEDER funds). The Digitized Sky Surveys were produced at STScI under U.S. Government Grant NAG W-2166.

\section{REFERENCES}

Acker, A., Marcout, J., Ochsenbein, F., et al. 1992, Strasbourg-ESO Catalogue of Galactic Planetary Nebulae. Part 1 and Part 2 (Germany: European Southern Observatory)

Acker, A., \& Neiner, C. 2003, A\&A, 403, 659

Akashi, M., Soker, N., \& Behar, E. 2006, MNRAS, 368, 1706

Akashi, M., Soker, N., Behar, E., \& Blondin, J. 2007, MNRAS, 375, 137

Aller, L. H. 1956, Gaseous Nebulae (London: Chapman and Hall)

Aryal, B., Rajbahak, C., \& Weinberger, R. 2010, MNRAS, 402, 1307

Balick, B., \& Frank, A. 2002, ARA\&A, 40, 439

Balick, B., Perinotto, M., Maccioni, A., Terzian, Y., \& Hajian, A. 1994, ApJ, 424,800

Bilíková, J., Chu, Y.-H., Gruendl, R. A., \& Maddox, L. A. 2010, AJ, 140, 1433

Blackman, E. G., Frank, A., Markiel, J. A., Thomas, J. H., \& Van Horn, H. M. 2001a, Nature, 409, 485

Blackman, E. G., Frank, A., \& Welch, C. 2001b, ApJ, 546, 288

Bond, H. E. 2000, in ASP Conf. Ser. 199, Asymmetrical Planetary Nebulae II: From Origins to Microstructures, ed. J. H. Kastner, N. Soker, \& S. Rappaport (San Francisco, CA: ASP), 115

Cahn, J. H., Kaler, J. B., \& Stanghellini, L. 1992, A\&AS, 94, 399

Cerruti-Sola, M., \& Perinotto, M. 1989, ApJ, 345, 339

Chu, Y.-H., Gruendl, R. A., \& Conway, G. M. 1998, AJ, 116, 1882

Chu, Y.-H., Guerrero, M. A., Gruendl, R. A., Williams, R. M., \& Kaler, J. B. 2001, ApJ, 553, L69

Chu, Y.-H., Kwitter, K. B., \& Kaler, J. B. 1993, AJ, 106, 650

Ciardullo, R., Bond, H. E., Sipior, M. S., et al. 1999, AJ, 118, 488

Ciardullo, R., Sigurdsson, S., Feldmeier, J. J., \& Jacoby, G. H. 2005, ApJ, 629, 499

Corradi, R. L. M., \& Schwarz, H. E. 1995, A\&A, 293, 871

Corradi, R. L. M., Sabin, L., Miszalski, B., et al. 2011, MNRAS, 410, 1349

Cox, P., Huggins, P. J., Maillard, J.-P., et al. 2002, A\&A, 384, 603

De Marco, O. 2009, PASP, 121, 316

De Marco, O., et al. 2011, in Asymmetrical Planetary Nebulae V Conference Proceedings, ed. O. De Marco, A. Frank, J. Kastner et al. (Manchester: Jodrell Bank Centre for Astrophysics)

Ercolano, B. 2009, MNRAS, 397, L69

Frankowski, A., \& Soker, N. 2009, ApJ, 703, L95

Freeman, P. E., Kashyap, V., Rosner, R., \& Lamb, D. Q. 2002, ApJS, 138, 185

Frew, D. J. 2008, PhD thesis, Macquarie Univ., NSW, Australia

Frew, D. J., Stanger, J., Fitzgerald, M., et al. 2011, PASA, 28, 83

Getman, K. V., Feigelson, E. D., Broos, P. S., Townsley, L. K., \& Garmire, G. P. 2010, ApJ, 708, 1760

Gruendl, R. A., Chu, Y.-H., O’Dwyer, I. J., \& Guerrero, M. A. 2001, AJ, 122, 308

Gruendl, R. A., Guerrero, M. A., Chu, Y.-H., \& Williams, R. M. 2006, ApJ, 653,339

Guerrero, M. A., Chu, Y.-H., \& Gruendl, R. A. 2000, ApJS, 129, 295

Guerrero, M. A., Chu, Y.-H., Gruendl, R. A., \& Meixner, M. 2005, A\&A, 430, L69

Guerrero, M. A., Chu, Y.-H., Gruendl, R. A., Williams, R. M., \& Kaler, J. B. 2001, ApJ, 553, L55

Guerrero, M. A., Gruendl, R. A., \& Chu, Y.-H. 2002, A\&A, 387, L1

Gurzadian, G. A. 1988, Ap\&SS, 149, 343

Herald, J. E., \& Bianchi, L. 2011, MNRAS, 417, 2440

Hoogerwerf, R., Szentgyorgyi, A., Raymond, J., et al. 2007, ApJ, 670, 442

Hook, I. M., Jørgensen, I., Allington-Smith, J. R., et al. 2004, PASP, 116, 425
Huarte-Espinosa, M., Frank, A., Balick, B., et al. 2012, MNRAS, in press (arXiv:1107.0415)

Jeffries, R. D., \& Stevens, I. R. 1996, MNRAS, 279, 180

Jones, D., Mitchell, D. L., Lloyd, M., et al. 2012, MNRAS, 420, 2271

Kastner, J. H. 2007, in Asymmetrical Planetary Nebulae IV, ed. R. L. M. Corradi, A. Manchado, \& N. Solur (La Palma: Instituto de Astrofisica de Canarias)

Kastner, J. H. 2009, in Protostellar Jets in Context, ed. K. Tsinganos, T. Ray, \& M. Stute (Berlin: Springer), 367

Kastner, J. H. 2011, in Asymmetric Planetary Nebulae V, ed. A. Zijlstra et al. (Manchester: Jodrell Bank Centre for Astrophysics)

Kastner, J. H., Balick, B., Blackman, E. G., et al. 2003, ApJ, 591, L37

Kastner, J. H., Gatley, I., Merrill, K. M., Probst, R., \& Weintraub, D. 1994, ApJ, 421,600

Kastner, J. H., Li, J., Vrtilek, S. D., et al. 2002, ApJ, 581, 1225

Kastner, J. H., Montez, R., Jr., Balick, B., \& De Marco, O. 2008, ApJ, 672, 957

Kastner, J. H., Soker, N., Vrtilek, S. D., \& Dgani, R. 2000, ApJ, 545, L57

Kastner, J. H., Vrtilek, S. D., \& Soker, N. 2001, ApJ, 550, L189

Kastner, J. H., Weintraub, D. A., Gatley, I., Merrill, K. M., \& Probst, R. G. 1996, ApJ, 462, 777

Kwok, S. 2000, the Origin and Evolution of Planetary Nebulae (New York: Cambridge University Press), 33

Kwok, S., Purton, C. R., \& Fitzgerald, P. M. 1978, ApJ, 219, L125

Li, S., Frank, A., \& Blackman, E. 2012, ApJ, 748, 24

Li, J., Kastner, J. H., Prigozhin, G. Y., et al. 2004, ApJ, 610, 1204

Longmore, A. J. 1977, MNRAS, 178, 251

Lou, Y.-Q., \& Zhai, X. 2010, MNRAS, 408, 436

Marten, H., \& Schönberner, D. 1991, A\&A, 248, 590

Mastrodemos, N., \& Morris, M. 1998, ApJ, 497, 303

Mendez, R. H., Herrero, A., \& Manchado, A. 1990, A\&A, 229, 152

Miszalski, B., Acker, A., Moffat, A. F. J., Parker, Q. A., \& Udalski, A. 2009a, A\&A, 496, 813

Miszalski, B., Acker, A., Parker, Q. A., \& Moffat, A. F. J. 2009b, A\&A, 505, 249

Miszalski, B., Corradi, R. L. M., Boffin, H. M. J., et al. 2011, MNRAS, 413, 1264

Monet, D. G., Levine, S. E., Canzian, B., et al. 2003, AJ, 125, 984

Montez, R., Jr. 2010, PhD thesis, Rochester Institute of Technology

Montez, R., Jr., De Marco, O., Kastner, J. H., \& Chu, Y.-H. 2010, ApJ, 721, 1820

Montez, R., Jr., Kastner, J. H., De Marco, O., \& Soker, N. 2005, ApJ, 635, 381 Morris, M. 1987, PASP, 99, 1115

Motch, C., Werner, K., \& Pakull, M. W. 1993, A\&A, 268, 561

Nordhaus, J., \& Blackman, E. G. 2006, MNRAS, 370, 2004

Nordhaus, J., Blackman, E. G., \& Frank, A. 2007, MNRAS, 376, 599

O'Dwyer, I. J., Chu, Y.-H., Gruendl, R. A., Guerrero, M. A., \& Webbink, R. F. 2003, AJ, 125, 2239

Parker, Q. A., Phillipps, S., Pierce, M. J., et al. 2005, MNRAS, 362, 689

Perinotto, M., Schönberner, D., Steffen, M., \& Calonaci, C. 2004, A\&A, 414, 993

Ressler, M. E., Cohen, M., Wachter, S., et al. 2010, AJ, 140, 1882

Reyes-Ruiz, M., \& López, J. A. 1999, ApJ, 524, 952

Ribeiro, T., \& Baptista, R. 2011, A\&A, 526, A150

Ruiz, N., Guerrero, M. A., Chu, Y.-H., \& Gruendl, R. A. 2011, AJ, 142, 91

Sahai, R., Kastner, J. H., Frank, A., Morris, M., \& Blackman, E. G. 2003, ApJ, 599, L87

Sahai, R., Morris, M. R., \& Villar, G. G. 2011, AJ, 141, 134

Sahai, R., \& Trauger, J. T. 1998, AJ, 116, 1357

Schmidt-Voigt, M., \& Koeppen, J. 1987, A\&A, 174, 211

Soker, N. 1997, ApJS, 112, 487

Soker, N., \& Kastner, J. H. 2002, ApJ, 570, 245

Soker, N., \& Livio, M. 1994, ApJ, 421, 219

Soker, N., Rahin, R., Behar, E., \& Kastner, J. H. 2010, ApJ, 725, 1910

Soker, N., \& Rappaport, S. 2000, ApJ, 538, 241

Stanghellini, L., Shaw, R. A., \& Villaver, E. 2008, ApJ, 689, 194

Steffen, M., Schönberner, D., \& Warmuth, A. 2008, A\&A, 489, 173

Stute, M., \& Sahai, R. 2006, ApJ, 651, 882

Su, K. Y. L., Chu, Y.-H., Rieke, G. H., et al. 2007, ApJ, 657, L41

Tarafdar, S. P., \& Apparao, K. M. V. 1988, ApJ, 327, 342

van Hoof, P. A. M., Barlow, M. J., Van de Steene, G. C., et al. 2011, arXiv: 1110.4524

Villaver, E., Manchado, A., \& García-Segura, G. 2002, ApJ, 581, 1204

Yu, Y. S., Nordon, R., Kastner, J. H., et al. 2009, ApJ, 690, 440

Zhekov, S. A., \& Perinotto, M. 1996, A\&A, 309, 648

Zijlstra, A. A., Lykou, F., McDonald, I., \& Lagadec, E. (ed.) 2011, Asymmetric Planetary Nebulae V (Manchester: Jodrell Bank Centre for Astrophysics) 